\title{
Stability and Capacity of Regular Wireless Networks
}

\author{
Gökhan Mergen, Student Member, IEEE, and Lang Tong, Fellow, IEEE
}

\begin{abstract}
We study the stability and capacity problems in regular wireless networks. In the first part of the paper, we provide a general approach to characterizing the capacity region of arbitrary networks, find an outer bound to the capacity region in terms of the transport capacity, and discuss connections between the capacity formulation and the stability of node buffers. In the second part of the paper, we obtain closed-form expressions for the capacity of Manhattan (two-dimensional grid) and ring networks (circular array of nodes). We also find the optimal (i.e., capacity-achieving) medium access and routing policies. Our objective in analyzing regular networks is to provide insights and design guidelines for general networks. The knowledge of the exact capacity enables us to quantify the loss incurred by suboptimal protocols such as slotted ALOHA medium access and random-walk-based routing. Optimal connectivity and the effects of link fading on network capacity are also investigated.
\end{abstract}

Index Terms-Capacity, multipacket reception, optimal connectivity, regular topology, scheduling, slotted ALOHA, stability, transport capacity, wireless networks.

\section{INTRODUCTION}

$\mathbf{T}$ HE network capacity problem deals with finding the fundamental limits on achievable communication rates in wireless networks. Computing the network capacity requires an optimization with respect to medium access and routing policies. For general networks, this task is typically prohibitive due to the excessive dimensionality of the problem. As a result, one has to contend with certain asymptotics and order computations (e.g., [1], [2]).

In this paper, we aim to provide an exception to this rule. Namely, we analyze certain regular networks for which we can compute the capacity explicitly. We obtain analytical expressions for the capacity, and find the leading coefficient besides the scaling law. The knowledge of the coefficient enables us to make comparisons between various design choices that affect the coefficient but not the scaling law. For example, we

Manuscript received January 23, 2003; revised December 20, 2004. This work was supported in part by the Multidisciplinary University Research Initiative (MURI) under the Office of Naval Research Contract N00014-00-1-0564 and the ARL CTA on Communications and Networks under Grant DAAD19-01-2-011. The material in this paper was presented in part at the 2001 Allerton Conference on Communications, Control, and Computing, Monticello, IL, October 2002; the 2002 Conference on Information Sciences and Systems, Princeton, NJ, March 2002, and the 2002 IEEE International Symposium in Information Theory, Lausanne, Switzerland, June/July 2002.

G. Mergen was with the School of Electrical and Computer Engineering, Cornell University, Ithaca, NY 14853 USA. He is now with Qualcomm, Campbell, CA 95008 USA (e-mail: mergen@ece.cornell.edu).

L. Tong is with the School of Electrical and Computer Engineering, Cornell University, Ithaca, NY 14853 USA (e-mail: ltong@ece.cornell.edu).

Communicated by L. Tassiulas, Associate Editor for Communication Networks.

Digital Object Identifier 10.1109/TIT.2005.847728

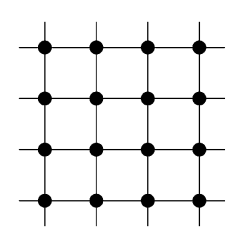

a)

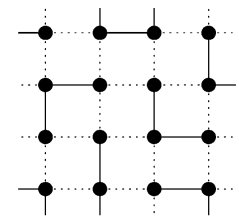

b)

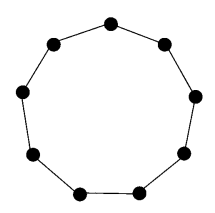

c)
Fig. 1. Examples of regular networks. (a) Manhattan. (b) Manhattan with fading links. (c) Ring.

quantify the loss incurred by suboptimal protocols and the effects of increasing connectivity. We also find the optimal (i.e., capacity-achieving) medium access and routing policies.

Before analyzing the capacity of regular networks, we provide a characterization of the capacity region of arbitrary networks. This characterization shows that every rate in the capacity region can be achieved by a class of policies that do randomized medium access and routing. Our formulation also suggests a natural outer bound on the capacity region in terms of the transport capacity of the network. To be able to consider regular networks, we extend the original definition of transport capacity [1] to networks with time-varying topology and arbitrary distance metric. The generalized transport capacity is used extensively in proving upper bounds on the capacity of regular networks.

The capacity formulation assumes that there are always packets waiting to be delivered at the source nodes. However, in reality, data packets arrive randomly in time, and the node buffers should be kept stable for proper network operation. Using a general network model with time-varying topology, we find a mild condition on the reception channel under which the proposed randomized policies stabilize the node buffers for all arrival rates in the capacity region.

The Manhattan network is a two-dimensional grid with size $\sqrt{N} \times \sqrt{N}$ (Fig. 1). Every node has four neighbors, and the nodes on the edge are connected to the nodes on the opposite edge forming a torus. We use the multipacket reception (MPR) channel model [3], which has been previously considered as an abstraction for code-division multiple access (CDMA) and multiple-antenna uplink. It is first shown that the capacity of the Manhattan network is

$$
\eta=\frac{K_{1}}{\sqrt{N}}+O\left(\frac{1}{N}\right)
$$

where the coefficient $K_{1}$ (given in Section IV) depends only on the channel reception capability. ${ }^{1}$ In case nodes can simultaneously receive multiple packets, $K_{1}$ increases but the form of $\eta$ does not change.

${ }^{1}$ When $f$ and $g$ are functions of $N$, we say that $f(N)=O(g(N))$ if there exists a scalar $C$ such that $|f(N)|<C g(N)$ for all $N$. 
Even though the capacity can be achieved by optimal medium access and routing, it is important to quantify the loss due to suboptimal, yet more practical, control policies. We analyze two extremes: a simple medium-access method (slotted ALOHA) and a simple routing protocol based on random walk. We show that the maximum achievable rate with slotted ALOHA medium access and optimal routing is

$$
\eta_{\mathrm{ALOHA}}=\frac{K_{2}}{\sqrt{N}}+O\left(\frac{1}{N}\right)
$$

where the coefficient $K_{2}$ (given in Section IV-B) is smaller than $K_{1}$. On the other hand, the achievable rate with optimal medium access and random-walk-based routing is shown to be $O(1 /(N \log N))$. These results suggest that the medium-access method in general does not change the order of the capacity, but the routing does change the order, and a poor routing protocol can significantly degrade the performance of large networks.

We next consider the case where the links in the Manhattan network are subject to time-varying fading (Fig. 1(b)). We use the collision channel model with a simple model for fading; links become ON or OFF randomly in each slot with probabilities $p, 1-p$, respectively. In case the control policy does not know the link states before making transmission decisions, we say that the policy is without link-state information (LSI). The capacity without LSI is shown to be

$$
\eta=\frac{K_{3}}{\sqrt{N}}+O\left(\frac{1}{N}\right)
$$

where the coefficient $K_{3}$ is given in Section V. We develop bounds on the capacity with LSI (denoted by $\eta^{\#}$ ). Namely, we show that the ratio $\eta^{\#} / \eta$ satisfies

$$
1 \leq \frac{\eta^{\#}}{\eta} \leq 2.86+O(1 / \sqrt{N})
$$

Furthermore, the bounds are tight in two extremes, i.e., $\eta^{\#} / \eta$ converges to 2.5 in the limit $p \rightarrow 0, N \rightarrow \infty$. Similarly, $\eta^{\#} / \eta$ converges to 1 in the limit $p \rightarrow 1, N \rightarrow \infty$. These bounds quantify the gain due to the knowledge of link state information.

Finally, we look at the optimal network-connectivity problem. Gupta and Kumar [1], and Gallager and Bertsekas ([4, p. 350]) discussed the tradeoff between throughput and connectivity, and argued that minimizing transmission radius while keeping the network connected leads to the highest throughput. Our analysis points out two cases where choosing minimal connectivity is not optimal. In the Manhattan network, we show that increasing connectivity from minimal to two-hop yields 54\% capacity increase if the nodes are capable of receiving eight or more packets simultaneously. Simultaneous receptions are particularly relevant for networks with spread spectrum and/or multiple antennas; in such systems, we expect performance gains from nonminimal connectivity. On the other hand, in ring networks (Fig. 1(c)), the capacity is shown to double by using higher connectivity. The capacity increase in the ring is not because of multipacket receptions, but because of the linear topology. These examples show that minimal connectivity is not always optimal, and there are potential benefits of higher connectivity depending on the channel usage and network topology.
Network capacity problems have been studied in several contexts. The early works focused on the computation of achievable rates with distributed protocols such as ALOHA (e.g., [5], [6], [4, p. 346]) and time-division multiple access (TDMA) (e.g., [7], [8]). Silvester and Kleinrock analyzed the capacities of regular networks with the slotted ALOHA protocol in [5], [6]. Our analysis of regular networks extend Silvester and Kleinrock's results in several directions considering centralized control as well as slotted ALOHA.

Gupta and Kumar [1] initiated a formal capacity analysis of random and arbitrary networks. Unlike most of the prior studies which started with a graph model having transmission powers fixed, Gupta and Kumar considered a joint optimization of transmission powers and schedules. They showed the fundamental result that the maximum per-node throughput scales roughly as $1 / \sqrt{N}$. For the regular networks in this paper, we provide the capacity coefficients besides the scaling law. These coefficients, which are not apparent in [1], reveal considerable insights into the design of medium-access (MAC) and optimal node connectivity.

More recently, a number of other works [2], [9]-[17] studied the capacity of wireless networks from various viewpoints. Toumpis and Goldsmith [9], [10] modeled the communication channel using deterministic rate matrices, and defined the notion of capacity region. They also analyzed the capacity regions of networks considering adaptive modulation and rates depending on the channel and interference conditions. Unlike Toumpis and Goldsmith, we also consider randomness in receptions. However, we do not consider rate adaptation.

The organization of the paper is as follows. In the next section, the network model and the multipacket reception channel are introduced. In Section III, we characterize the capacity region of arbitrary networks, and discuss its connections with the transport capacity and network stability. In Section IV, we compute the capacity of Manhattan networks and find capacityachieving MAC and routing policies. The maximum achievable rate with slotted ALOHA is also computed. In Section V, the capacity with fading is analyzed. In Section VI, optimal connectivity in Manhattan and ring networks is investigated. Section VII concludes the paper.

\section{NETWORK MODEL}

We consider networks represented with an undirected graph such that two nodes $i$ and $j$ can communicate directly only if they are connected with an edge. The graph models are traditionally used with the collision channel assumption [18]. That is, two nodes can communicate directly if they are within a distance $r$, and transmission from node $i$ to node $j$ is successful if there is no other transmitter within distance $r$ to node $j$ (see Fig. 2).

In wireless networks with CDMA and/or multiple antennas, the collision channel assumptions do not hold. That is because the nodes might be capable of receiving multiple packets simultaneously, and there may be unexpected reception errors due to channel time variation. To be able to consider such networks, we use the graph models with MPR [3], [19]. Suppose that nodes cannot transmit and receive at the same time. Each node can 


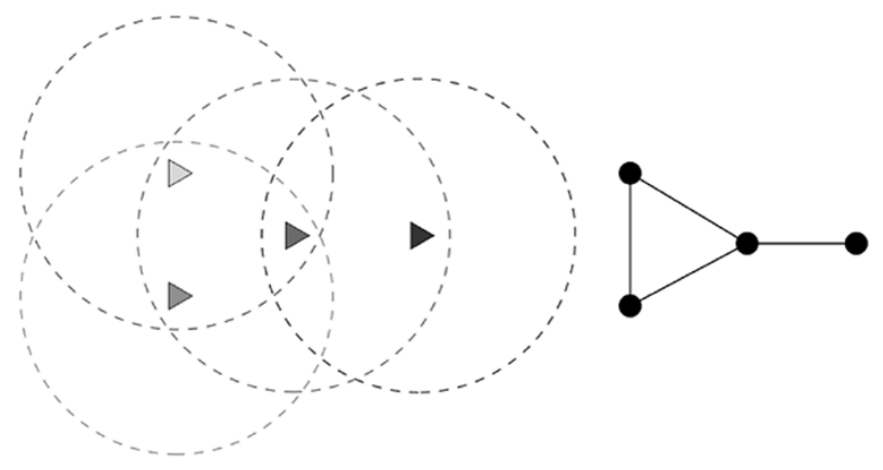

Fig. 2. Nodes and transmission ranges in a planar network are shown on the left. The right figure shows the corresponding graph.

transmit at most one packet at a time. In each slot, a node can correctly receive a fraction of the number of transmissions in its neighborhood. The reception probabilities are given by the receiver MPR matrix $\boldsymbol{C}$. The entries of the MPR matrix are given by

$C_{n, k}=\operatorname{Pr}\{k$ packets are received $\mid n$ packets are transmitted in the neighborhood\}.

The receiver MPR matrix $\boldsymbol{C}$ is defined by

$$
\boldsymbol{C}=\left(\begin{array}{cccc}
C_{1,0} & C_{1,1} & & \\
C_{2,0} & C_{2,1} & C_{2,2} & \\
\vdots & \vdots & \vdots & \ddots
\end{array}\right)
$$

Given the transmitting nodes in the network, the successful reception events at different receivers are assumed independent. Each transmitter around a given receiver has equal chance for getting successfully received.

The MPR matrix, in general, depends on the channel characteristics, the type of modulation used, and the multiuser detection/equalization method receivers apply. A variety of physical layers have been modeled using MPR and its variants (e.g., CDMA [20]-[22] and multiple antenna uplink [23], [24]). Some simple examples of MPR are the collision channel $\boldsymbol{C}_{1}$ and the 2-collision channel $\boldsymbol{C}_{2}$

$\boldsymbol{C}_{1}=\left(\begin{array}{ccccc}0 & 1 & & & \\ 1 & 0 & 0 & & \\ 1 & 0 & 0 & 0 & \\ \vdots & \vdots & \vdots & \vdots & \ddots\end{array}\right), \quad \boldsymbol{C}_{2}=\left(\begin{array}{ccccc}0 & 1 & & & \\ 0 & 0 & 1 & & \\ 1 & 0 & 0 & 0 & \\ \vdots & \vdots & \vdots & \vdots & \ddots\end{array}\right)$.

As a generalization of $\boldsymbol{C}_{1}$ and $\boldsymbol{C}_{2}$, we define the $M$-collision channel $\boldsymbol{C}_{M}$ in which simultaneous reception of less than or equal to $M$ packets is possible; if more then $M$ packets are transmitted, then none of them are correctly received.

A motivation for considering the $M$-collision channel comes from the CDMA with matched filter. In the common SINR (signal-to-interference-plus-noise ratio) threshold model for CDMA [25], a transmitter is considered successful if its power divided by the noise plus interference exceeds a certain threshold. If the transmission and reception powers of the nodes are identical, then the SINR threshold model is equivalent to the $M$-collision channel for some $M$ proportional to the spreading gain. The $C_{M}$ has also been previously considered in [20], [26], [27].

\section{CAPACITY REgION OF ARbitRARY NETWORKS}

In this section, we provide a characterization of the capacity region of arbitrary networks. We also provide connections of the capacity region with the notion of transport capacity and the stability of node buffers in a network with random packet arrivals.

Let $\mathcal{G}=(\mathcal{N}, \mathcal{L})$ be an undirected graph ${ }^{2}$ with nodes $\mathcal{N}=$ $\{0, \ldots, N-1\}$ and the set of links $\mathcal{L}$. Suppose that every node holds infinitely many packets waiting to be delivered. Node $i$ wishes to communicate with node $j$ with rate $\lambda_{i j}$ [packets/slot]. A rate vector $\left(\lambda_{i j}: i, j \in \mathcal{N}\right)$ is called achievable if there exists a scheduling policy that can guarantee those rates, i.e.,

$$
\liminf _{T \rightarrow \infty} \frac{1}{T} \sum_{t=0}^{T-1} W_{i j}(t) \geq \lambda_{i j}, \quad \forall i, j \in \mathcal{N}
$$

almost surely, where $W_{i j}(t)$ denotes the number of packets with source $i$ and destination $j$ delivered in slot $t$. The closure of the set of achievable rate vectors is called the capacity region of the network.

\section{A. Characterization of the Capacity Region}

In this paper, we will be interested in a particular class of scheduling policies that do randomized routing and medium access. These will be called randomized time-division (RTD) policies for reasons that will become apparent. Three mechanisms used in RTD policies are the following.

i) Routing: Let $\mathrm{P}_{i j}$ denote the set of all noncyclic paths from node $i$ to node $j$. The routing in RTD policies is done such that every packet to be delivered from source $i$ to destination $j$ is assigned a random route $P \in \mathrm{P}_{i j}$ according to a certain probability distribution $\left\{x_{P}\right\}_{P \in \mathrm{P}_{i j}}$.

ii) Medium Access: Each schedule is denoted by a subset $\mathcal{E}$ of the set $\mathcal{L}$. We call a schedule $\mathcal{E}$ admissible if no transmitter is repeated twice. The set of admissible schedules is denoted by E. RTD policies control the transmissions such that a random schedule $\mathcal{E}$ is chosen in each slot according to a probability distribution $\{p(\mathcal{E})\}_{\mathcal{E} \in \mathrm{E}}$.

iii) Queuing Discipline: If a node is scheduled to transmit over link $l$, a route $P \in \mathrm{P}_{i j}$ (such that $l \in P$ ) is chosen randomly with probability

$$
Q(P, l):=\frac{x_{P} \lambda_{i j}}{\sum_{i, j \in \mathcal{N}} \sum_{P \in \mathrm{P}_{i j}: l \in P} x_{P} \lambda_{i j}} .
$$

If the scheduled node has any packet with route $P$, it is transmitted; otherwise, the node can transmit any other packet in its queue. Equation (2) assures fairness, since each route passing through link $l$ is allocated bandwidth proportional to its traffic rate $x_{P} \lambda_{i j}$.

${ }^{2}$ The set $\mathcal{L}$ consists of ordered node pairs $(i, j)$. A graph $(\mathcal{N}, \mathcal{L})$ is called undirected if $(i, j) \in \mathcal{L}$ implies $(j, i) \in \mathcal{L}$. 
Remark: An RTD policy is specified by the probability distributions $\left\{p(\mathcal{E}\}_{\mathcal{E} \in \mathrm{E}},\left\{x_{P}\right\}_{P \in \mathrm{P}_{i j}}, \forall i \neq j\right.$, and a set of target rates $\left(\lambda_{i j}: i, j \in \mathcal{N}\right)$. We use the term "randomized time-division," because the RTD policies essentially apply a randomized version of TDMA.

Next, we will find the set of achievable rates with the RTD policies. Let $\Pi(l ; \mathcal{E})$ denote the probability of successful transmission over link $l=(i, j)$ given that schedule $\mathcal{E}$ is applied. This probability is equal to zero if $l \notin \mathcal{E}$ or if $j$ is scheduled to transmit as well (recall that nodes cannot transmit and receive simultaneously). Otherwise

$$
\Pi(l ; \mathcal{E})=\frac{\sum_{k=1}^{n} k C_{n, k}}{n}:=\frac{C_{n}}{n}
$$

where $n$ is the total number of transmitters around node $j$ in schedule $\mathcal{E}$ (this number includes both the transmissions intended for $j$ and the transmissions that are not). To see (3), recall that $C_{n, k}$ is the probability that $k$ out of $n$ transmitters are successful. Node $i$ belongs to the set of successful transmitters with probability $k / n$, since every size- $k$ subset is equally likely to succeed. Summation of $(k / n) C_{n, k}$ yields (3). Here, it is important to note that the $C_{n}$ itself has a physical meaning; it is the expected number of successful receptions at a node hearing $n$ packets.

The next theorem characterizes the capacity region of the network. It also asserts that RTD policies are sufficient to achieve every rate in the capacity region.

Theorem 1: A rate vector $\left(\lambda_{i j}: i, j \in \mathcal{N}\right)$ is achieved by an RTD policy if

$$
\sum_{i, j \in \mathcal{N}} \sum_{P \in \mathrm{P}_{i j}: l \in P} x_{P} \lambda_{i j} \leq \sum_{\mathcal{E} \in \mathrm{E}} \Pi(l ; \mathcal{E}) p(\mathcal{E}), \quad \forall l \in \mathcal{L}
$$

i.e., if the aggregate traffic over link $l$ is less than the throughput over link $l$ for all $l$. For given rate vector $\left(\lambda_{i j}: i, j \in \mathcal{N}\right)$, if there does not exist an RTD policy satisfying (4), then this rate cannot be achieved by any policy. Hence, the capacity region is equal to the union of all $\left(\lambda_{i j}: i, j \in \mathcal{N}\right)$ satisfying (4) with some RTD policy.

Proof: The proof is omitted for brevity. See [28] for the proof of an extended version of the theorem for a general reception model where the reception probabilities are not necessarily symmetric, and the network topology is possibly time varying.

\section{B. Transport Capacity}

In this subsection, we extend the original definition of transport capacity [1] to networks with probabilistic receptions. This extension allows us to handle the transport capacity in a more general setting where the distance metric is not Euclidean.

Let $d(i, j)$ denote the distance between two nodes $i$ and $j$. Equivalently, the notation $d(l)$ will be used for $l=(i, j)$. The distance metric is assumed to satisfy the triangle inequality, i.e.,

$$
d(i, j) \leq \sum_{l \in P} d(l), \quad \forall P \in \mathrm{P}_{i j} .
$$

Finding whether a rate vector is achievable requires checking the existence of medium access and routing probabilities satisfying (4). This task is generally very challenging for large net- works. The next proposition provides a necessary condition for achievability which is relatively easier to check.

Proposition 1: If rate vector $\left(\lambda_{i, j}: i, j \in \mathcal{N}\right)$ is in the capacity region, then

$$
\sum_{i, j \in \mathcal{N}} \lambda_{i j} d(i, j) \leq \max _{\mathcal{E} \in \mathrm{E}} \sum_{l \in \mathcal{L}} d(l) \Pi(l ; \mathcal{E}) .
$$

Remark: The proposition is easier to understand if one views $\sum_{i, j} \lambda_{i j} d(i, j)$ as the work (i.e., the rate-distance product) needed to achieve rate vector $\left\{\lambda_{i j}\right\}$. We call the right-hand side of (5) as the transport capacity of the network, i.e., the maximum rate-distance product achieved by any schedule. Equation (5) provides a simple outer bound to the capacity region.

Proof: Let $\left\{\lambda_{i j}\right\}$ be achievable with some $\{p(\mathcal{E})\}$ and $\left\{x_{P}\right\}$. Then

$$
\begin{aligned}
\sum_{i, j \in \mathcal{N}} \lambda_{i j} d(i, j) & =\sum_{i, j \in \mathcal{N}} \sum_{P \in \mathrm{P}_{i j}} x_{P} \lambda_{i j} d(i, j) \\
& \leq \sum_{i, j \in \mathcal{N}} \sum_{P \in \mathrm{P}_{i j}} \sum_{l \in P} x_{P} \lambda_{i j} d(l) \\
& =\sum_{l \in \mathcal{L}} d(l) \sum_{i, j \in \mathcal{N}} \sum_{P \in \mathrm{P}_{i j}: l \in P} x_{P} \lambda_{i j} \\
& \leq \sum_{l \in \mathcal{L}} d(l) \sum_{\mathcal{E} \in \mathrm{E}} \Pi(l ; \mathcal{E}) p(\mathcal{E}) \\
& \leq \max _{\mathcal{E} \in \mathrm{E}} \sum_{l \in \mathcal{L}} d(l) \Pi\left(l ; \mathcal{E}_{v}, v\right)
\end{aligned}
$$

where (6) follows from the triangle inequality, and (7) is because of achievability.

The above proposition holds for any distance metric satisfying the triangle inequality. The conventional definition of metric imposes the additional constraints of nonnegativity and symmetry of $d(i, j)$ (e.g., [29]). As is obvious from the proof, these constraints are not needed for the above result.

Definition: Rate $\lambda \geq 0$ is called uniformly achievable if the rate vector $\left(\lambda_{i j}=\lambda: i \neq j, i, j \in \mathcal{N}\right)$ is achievable. The network capacity, denoted by $\eta$, is defined as the maximum uniformly achievable rate multiplied by $N-1$. This can be viewed as the maximum per-node throughput.

The following theorem provides an upper bound to the network capacity in terms of transport capacity.

Theorem 2: The network capacity satisfies

$$
\eta \leq \frac{1}{\bar{L} N} \max _{\mathcal{E} \in \mathrm{E}} \sum_{l \in \mathcal{L}} d(l) \Pi(l ; \mathcal{E})
$$

where $\bar{L}$ is the average distance between two arbitrarily selected nodes, i.e.,

$$
\bar{L}=\frac{1}{N(N-1)} \sum_{i, j \in \mathcal{N}} d(i, j) .
$$

Proof: Substitute $\lambda_{i j}=\lambda$ in (5). After rearranging (5), observe that $(N-1) \lambda$ is less than the right-hand side of $(8)$ for all $\lambda$. Notice that this result requires $\bar{L}$ to be positive. 
The MPR model can be generalized in two ways. First, one can consider asymmetric reception probabilities, which is the case in reality, since different users have different channel strengths. Second, the reception probabilities may change over time if the network topology and/or user channel strengths are time varying. A general network model with asymmetric and time-varying reception probabilities is introduced in Appendix A. Interestingly, Theorems 1 and 2 can be generalized to the general model with minor changes. The network capacity with time-varying reception probabilities is shown to be upper-bounded by the transport capacity averaged over different network states.

\section{Stability of Node Buffers}

In the capacity analysis, it is assumed that there are always packets to be delivered at the source nodes. However, in reality, the data packets arrive randomly in time, and for proper network operation the node buffers should be kept stable. In this section, we argue that the achievability in a backlogged network implies the stability of node buffers provided that the reception channel satisfies a mild condition.

Consider a network with graph $\mathcal{G}=(\mathcal{N}, \mathcal{L})$. Suppose that packets with source $i$ and destination $j$ arrive at node $i$ according to an ergodic and stationary process with rate $\lambda_{i j}$. Arrival processes for different $i, j$ are assumed independent. Each node has an infinite buffer to hold packets, and the network starts operation with empty buffers.

The question we are interested in is: Is there a scheduling policy stabilizing the node buffers for given arrival processes with rate vector $\left(\lambda_{i j}: i, j \in \mathcal{N}\right)$ ?

Definition: Let $Q_{i}(t)$ be the number of packets in node $i$ 's buffer at time $t$. The buffer of node $i$ is called stable if

$$
\lim _{B \rightarrow \infty} \limsup _{t \rightarrow \infty} \operatorname{Pr}\left\{Q_{i}(t)>B\right\}=0 .
$$

The network is called stable if all node buffers are stable; otherwise, it is unstable. The stability region is the closure of the set of arrival rates $\left(\lambda_{i j}: i, j \in \mathcal{N}\right)$ for which the network can be stabilized with a scheduling policy.

This notion of stability admits the following heuristic interpretation. Supposing $B$ is the buffer capacity of the node, one can view $\limsup _{t \rightarrow \infty} \operatorname{Pr}\left\{Q_{i}(t)>B\right\}$ as the asymptotic buffer overflow probability of the $i$ th queue. This queue is called stable if its asymptotic buffer overflow probability goes to zero as the buffer size $B$ goes to infinity. Equation (9) was first used by Tsybakov and Bakirov in the context of stability of slotted ALOHA. Depending on the network model, other stability notions are also used in the literature (e.g., [30]-[34]).

Next, we will provide the condition for achievability to imply stability. Let $\mathcal{F}$ be a subset of links in a schedule $\mathcal{E}$. Let $\Pi(\mathcal{F} ; \mathcal{E})$ be the probability of the event that all links in $\mathcal{F}$ are successful given that the schedule $\mathcal{E}$ is used. For all $\mathcal{F}, \mathcal{E}, \mathcal{E}^{\prime} \in \mathrm{E}$ satisfying $\mathcal{F} \subset \mathcal{E} \subset \mathcal{E}^{\prime}$, we require the channel to satisfy

$$
\Pi\left(\mathcal{F} ; \mathcal{E}^{\prime}\right) \leq \Pi(\mathcal{F} ; \mathcal{E})
$$

i.e., the probability of success should decrease when there are more transmissions. This condition is naturally satisfied in practical communication systems. However, it is not met by some MPR matrices, and needs to be specified for mathematical precision.

Theorem 3: The network capacity and the stability regions are identical if the reception probabilities satisfy (10). In particular, let $\left(\lambda_{i j}: i, j \in \mathcal{N}\right)$ be a rate vector for which the achievability condition (4) is satisfied by an RTD policy with strict inequalities. Then, the RTD policy also stabilizes the network with arrival rate $\left(\lambda_{i j}: i, j \in \mathcal{N}\right)$.

Proof: See [28]. In the proof, we first show network stability with an RTD policy under the assumption that all nodes are backlogged. Then, inequality (10) is used to show that the queue lengths in the backlogged network stochastically dominates the queue lengths in normal network. Hence, the stability of backlogged network implies the stability of normal network. Reference [28] also gives a counter example showing that the stability and the capacity regions are not necessarily the same when condition (10) does not hold.

See Appendix A, for an extension of Theorem 3 to networks with time-varying topology and asymmetric reception probabilities.

\section{CAPACiTy of Manhattan Networks}

In this section, we find the capacity of regular networks. Before going into the details, we first outline our methodology. Theorem 2 is our main tool for upper-bounding the capacities of regular networks. In order to apply Theorem 2, we will compute the average path length $\bar{L}$ and the transport capacity. After finding an appropriate upper bound on the network capacity, we will show that the upper bound is achieved exactly, or approximately with an error of order $O(1 / N)$ (or, with an error $O\left(1 / N^{2}\right)$ in ring networks). Specifically, we will find routing and scheduling probabilities such that the corresponding RTD policy achieves the upper bound. The basic idea behind optimal routing in regular networks is to use the shortest paths while balancing the routing load. On the other hand, we will see that the optimal MAC problem is equivalent to packing the maximum number of transmissions into a regular lattice.

A node in the Manhattan network is determined by two coordinates $(x, y) \in\{0, \ldots, \sqrt{N}-1\} \times\{0, \ldots, \sqrt{N}-1\}$. We define the distance between two nodes $\left(x_{0}, y_{0}\right)$ and $\left(x_{1}, y_{1}\right)$ as the minimum number of hops to reach from one node to another, i.e.,

$$
\begin{aligned}
d\left\{\left(x_{0}, y_{0}\right),\left(x_{1}, y_{1}\right)\right\} & \\
= & \min \{\delta x, \sqrt{N}-\delta x\}+\min \{\delta y, \sqrt{N}-\delta y\}
\end{aligned}
$$

where $\delta x=\left|x_{0}-x_{1}\right|$ and $\delta y=\left|y_{0}-y_{1}\right|$. Recall that the nodes on one edge of the Manhattan network are connected to the nodes on the opposite edge; because of this property, the distance metric is defined as (11) instead of $d\left\{\left(x_{0}, y_{0}\right),\left(x_{1}, y_{1}\right)\right\}=$ $\delta x+\delta y$. A simple calculation yields the following proposition.

Proposition 2: In the Manhattan network with $N$ nodes, the average distance between two nodes $\bar{L}$ is given by

$$
\bar{L}=\left\{\begin{array}{ll}
\frac{\sqrt{N}}{2}, & \sqrt{N} \text { odd } \\
\frac{N \sqrt{N}}{2(N-1)}, & \sqrt{N} \text { even }
\end{array}=\frac{\sqrt{N}}{2}+O(1 / \sqrt{N}) .\right.
$$

Proof: See Appendix B. 


\begin{tabular}{c|c|c|c|c}
\multicolumn{7}{c}{ TABLE I } \\
\multicolumn{5}{c}{$e_{\tau}$ VERSUS $\tau$} \\
\hline & $\tau=1$ & $\tau=2$ & $\tau=3$ & $\tau=4$ \\
\hline$e_{\tau}$ & 4 & 3 & 4 & 5 \\
\hline
\end{tabular}

The following lemma will be used to show the achievability of the capacity of Manhattan networks.

Lemma 1: In the Manhattan network with uniform traffic $\lambda$, there exists routing probabilities $\left\{x_{P}\right\}$ such that the traffic over any link $l$ is $\lambda(N-1) \bar{L} / 4$.

Proof: See Appendix C.

Lemma 1 is a load-balancing property; it guarantees the existence of a routing protocol that distributes the traffic load over the links uniformly. In its proof, we show that every symmetric, shortest path routing satisfies the desired property. Because of using the shortest paths, the quantity in Lemma 1 is the minimum load that has to be put over the links to achieve $\lambda$.

The next theorem characterizes the capacity of Manhattan networks.

Theorem 4: (Capacity of Manhattan Networks): Let $\eta$ be the capacity of a Manhattan network with $N$ nodes each with MPR matrix $C$. Define

$$
\eta^{*}=\max _{i=1, \ldots, 4} \frac{C_{i}}{i+1} \frac{1}{\bar{L}}
$$

The following relations hold:

$$
\begin{aligned}
& \eta \leq \eta^{*} \\
& \eta=\eta^{*}+O\left(\frac{1}{N}\right) .
\end{aligned}
$$

Furthermore, if $\sqrt{N}$ is divisible by $e_{\tau}$, then $\eta=\eta^{*}$, where

$$
\tau=\underset{i=1, \ldots, 4}{\arg \max } \frac{C_{i}}{i+1}
$$

and $e_{\tau}$ is given in Table $\mathrm{I}$.

Proof: First, we will argue that

$$
\frac{1}{\bar{L} N} \sum_{l \in \mathcal{L}} \Pi(l ; \mathcal{E}) \leq \eta^{*}
$$

for every transmission set $\mathcal{E}$. The distance between two neighboring nodes is 1 , and the previous inequality proves $\eta \leq \eta^{*}$ as a result of Theorem 2 .

To see (14), we will classify the nodes in the network according to the transmission set $\mathcal{E}$. Every node either transmits a packet or stays in the reception mode. Every node $k$ in the reception mode receives two types of packets: the packets intended for the receiver $k$ and the packets intended for other nodes. Let $\mathcal{A}_{i j}$ be the set of all nodes that do not transmit, receive $j$ packets for itself, and receive $i-j$ packets transmitted for other nodes (see Fig. 3). Define $A_{i j}=\left|\mathcal{A}_{i j}\right|$ as the number nodes in $\mathcal{A}_{i j}$. Note that $A_{i j}$ can be nonzero only for $0 \leq j \leq i \leq 4$, since nodes can receive packets from at most four other nodes. Every node in the network can transmit one packet at a time and for every receiver in set $\mathcal{A}_{i j}$ there exists $j$ other transmitters in the network. The $A_{i j}$ must satisfy

$$
\sum_{i=0}^{4} \sum_{j=0}^{i}(1+j) A_{i j} \leq N
$$

since the total number of nodes in the network is $N$.

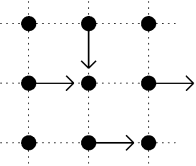

(a)

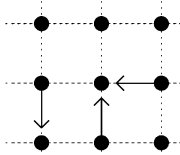

(b)

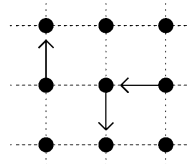

(c)
Fig. 3. Figures illustrate the definition of $\mathcal{A}_{i j}$. In (a), the node in the center receives two packets intended for itself, and two packets intended for other nodes; therefore, it is an element of the set $\mathcal{A}_{4,2}$. The node in the center in (b) receives two packets for itself, and one packet intended for some other node; it is in $\mathcal{A}_{3,2}$. In (c), the node in the center is not an element of any $\mathcal{A}_{i, j}$, since it is transmitting.

Each transmission to a node hearing $i$ packets becomes successful with probability $C_{i} / i$ (recall (3)). Therefore,

$$
\frac{1}{\bar{L} N} \sum_{l \in \mathcal{L}} \Pi(l ; \mathcal{E})=\frac{1}{\bar{L} N} \sum_{i=0}^{4} \sum_{j=0}^{i} \frac{j}{i} C_{i} A_{i j} .
$$

Consider the optimization problem

$$
\begin{array}{ll}
\text { maximize } & \xi=\frac{1}{\bar{L} N} \sum_{i=0}^{4} \sum_{j=0}^{i} \frac{j}{i} C_{i} A_{i j} \\
\text { subject to } & \sum_{i=0}^{4} \sum_{j=0}^{i}(1+j) A_{i j} \leq N \\
& A_{i j} \geq 0
\end{array}
$$

where the maximization is with respect to real-valued $A_{i j}$. In the original problem, the $A_{i j}$ can only take integer values. Since we relax this constraint (and some others), the solution of the above optimization yields an upper bound on (15).

Equation (16) is a linear programming problem, and its solution is well known to be at one of the extreme points of the constraint set. Since the constraint set is a simplex, the solution is attained at one of its corners

$$
A_{i j}= \begin{cases}\frac{N}{j^{\prime}+1}, & \text { if } i=i^{\prime}, j=j^{\prime} \\ 0, & \text { otherwise }\end{cases}
$$

for some $0 \leq j^{\prime} \leq i^{\prime} \leq 4$. When we substitute the possible candidates for $A_{i j}$ in (16), it is seen that

$$
\begin{aligned}
\xi & =\frac{1}{\bar{L} N} \sum_{i=0}^{4} \sum_{j=0}^{i} \frac{j}{i} C_{i} A_{i j} \\
& =\frac{1}{\bar{L}} \frac{j^{\prime}}{i^{\prime}\left(j^{\prime}+1\right)} C_{i^{\prime}} \\
& \leq \frac{1}{\bar{L}} \frac{C_{i^{\prime}}}{i^{\prime}+1} \\
& \leq \eta^{*} .
\end{aligned}
$$

The inequality before (17) is true because $j^{\prime} /\left(j^{\prime}+1\right)$ is an increasing function of $j^{\prime} \leq i^{\prime}$. Thus, (14) holds and $\eta^{*}$ is an upper bound to the network capacity.

Next, we will show that $\eta^{*}+O(1 / N)$ is achievable. For this, we will use the following medium access policy, which will be called the $\tau$-MPR scheduling. The $\tau$ used to achieve $\eta^{*}+$ $O(1 / N)$ is defined in (13). In $\tau$-MPR scheduling, the network is tiled using the $\tau$-MPR pattern (see Fig. 4) and its shifted/rotated versions. Every scheduled receiver receives $\tau$ packets intended for itself. The network is tiled perfectly with the $\tau$-MPR patterns if and only if $\sqrt{N}$ is divisible by $e_{\tau}$ (Table I). 


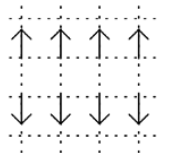

a)

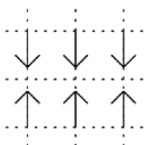

b)

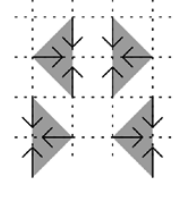

c)

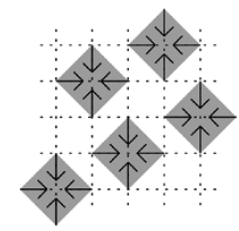

d)
Fig. 4. Different scheduling patterns for $\tau$-MPR, $\tau \in\{1,2,3,4\}$. Dashed lines are the links, and the arrows are scheduled packet transmissions. (a) 1 -MPR $4 \times 4$ nodes, (b) 2 -MPR $3 \times 3$ nodes, (c) 3 -MPR $4 \times 4$ nodes, (d) 4 -MPR $5 \times 5$ nodes.

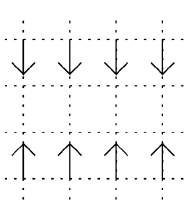

(a)

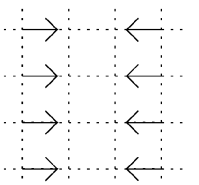

(e)

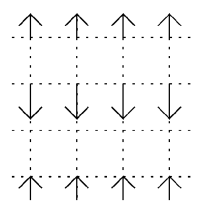

(b)

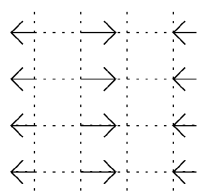

(f)

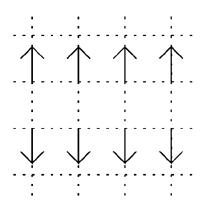

(c)

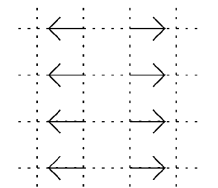

(g)

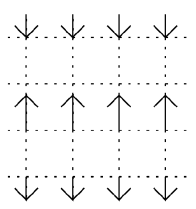

(d)
Fig. 5. Eight phases of the 1-MPR scheduling in a network with 16 nodes. In each slot, the medium access protocol applies a randomly selected phase with $1 / 8$ probability.

To demonstrate the use of $\tau$-MPR scheduling, suppose that $\tau=1$ and $\sqrt{N}$ is divisible by 4 . In this case, 1 -MPR pattern and its shifted/rotated versions (Fig. 5) can tile all of the network. We call each shifted/rotated version of 1-MPR pattern as a phase of 1 -MPR scheduling. For medium access, we assign $1 / 8$ probability to each phase. With this assignment of probabilities every node gets a chance to transmit to each neighbors with probability $1 / 8$. Each transmission becomes successful with probability $C_{1}$. As a result, links can deliver $C_{1} / 8$ throughput over each direction. Supposing that 1-MPR scheduling is used together with the routing protocol provided by Lemma 1, all rates $\lambda$ satisfying

$$
\frac{(N-1) \lambda \bar{L}}{4} \leq \frac{C_{1}}{8}
$$

are uniformly achievable. This shows that $\lambda=\eta^{*} /(N-1)$ is uniformly achievable.

Using identical arguments, it can be seen that $\lambda=\eta^{*} /(N-1)$ is achieved uniformly by $\tau$-MPR scheduling whenever $\sqrt{N}$ is divisible by $e_{\tau}$. In case $\sqrt{N}$ is not divisible by $e_{\tau}$, all the network except a small portion can be tiled. In general, the number of nodes which can be scheduled with $\tau$-MPR is $N+O(\sqrt{N})$. Again, by using shifted/rotated versions of the $\tau$-MPR

$$
\frac{1}{4}\left[1+O\left(\frac{1}{\sqrt{N}}\right)\right] \max _{i=1, \ldots, 4} \frac{C_{i}}{(i+1)}
$$

traffic can be supported over each link. The $O(1 / \sqrt{N})$ factor in (19) is negative, and it decreases the link capacities. Therefore, $\eta^{*}$ is not achievable in general, but $\eta^{*}+O(1 / N)$ can be achieved. The achievability part of the theorem follows.
Remark: Note that $\tau$-MPR is the schedule that maximizes the total number of successful transmissions in the network if $e_{\tau}$ divides $\sqrt{N}$. In $\tau$-MPR, there are $N /(\tau+1)$ receivers in the network each receiving $\tau$ packets. Thus, the total number of successful transmissions is $N C_{\tau} /(\tau+1)$. This quantity divided by $\bar{L} N$ is shown to upper-bound the $\xi$ in (17). Hence, $\tau$-MPR maximizes $\xi$ and, equivalently, maximizes the total number of successful transmissions in the network.

\section{A. Random Routing}

In large networks, topology discovery may not be feasible, and nodes may not be able to use shortest routes. Similarly, during network initialization nodes spend some time discovering the network and may not be able to use the optimal routes. Gossiping [35], flooding, and random-walk-based routing [36] are alternatives that do not require the nodes to know the whole network topology. In random-walk-based routing, the packets are relayed at each consecutive hop to a randomly chosen neighbor with uniform probabilities. If the network is connected, every packet eventually reaches its destination, although the delivery may take a long time. In the proof showing the achievability of $\eta$ we used the shortest path routing, which gives an average path length $\bar{L}$ proportional to $\sqrt{N}$. In [37], [38], it is shown that the average path length with random walk is of the order $N \log N$. Using an argument similar to the one in Theorem 4 , it is easily seen that the maximum achievable rate with random-walk-based routing is $O(1 /(N \log N))$, whereas the capacity scales as $(1 / \sqrt{N})$. Similarly, each flooding requires $N$ transmissions, resulting in $O(1 / N)$ throughput scaling. These results show that the cost of lacking (or not using) topology information can be very high in large networks.

\section{B. Capacity With Slotted ALOHA}

In a distributed wireless network, topology-specific scheduling may not be implementable in practice. On the other hand, it is important to quantify the performance loss due to suboptimal, but easily implementable MAC protocols such as slotted ALOHA. In the next theorem we will give the highest rate achievable with the slotted ALOHA. We consider the capacity setup where every node has infinitely many packets waiting in its queue to be delivered to the other nodes. Every node randomly and independently makes a transmission decision in each slot; a node chooses to transmit a packet with transmission probability $q$, the neighbor to be transmitted is chosen with uniform probabilities.

Theorem 5: (Capacity With Slotted ALOHA): The maximum uniformly achievable rate with slotted ALOHA is given by $\left(\eta_{\text {ALOHA }} / N-1\right)$, where

$$
\eta_{\mathrm{ALOHA}}=\frac{1}{4 \bar{L}} \max _{0 \leq q \leq 1} \sum_{k=1}^{4}\left(\begin{array}{l}
4 \\
k
\end{array}\right) q^{k}(1-q)^{5-k} C_{k} .
$$

Proof: With transmission probability $q$, each node receives

$$
R(q)=\frac{1}{4} \sum_{k=1}^{4}\left(\begin{array}{l}
4 \\
k
\end{array}\right) q^{k}(1-q)^{5-k} C_{k}
$$


TABLE II CAPACITY VERSUS SLOTTED ALOHA CAPACITY

\begin{tabular}{c|c|c|c|c}
\hline & $M=1$ & $M=2$ & $M=3$ & $M=4$ \\
\hline$\eta \cdot \sqrt{N}$ & 1.00 & 1.33 & 1.50 & 1.60 \\
\hline$\eta_{A L O H A} \cdot \sqrt{N}$ & 0.16 & 0.34 & 0.46 & 0.50 \\
\hline
\end{tabular}

intended packets for itself on the average. To see this, observe that each node stays in reception mode with probability $(1-q)$. A node in the reception mode receives

$$
\sum_{k=1}^{4}\left(\begin{array}{l}
4 \\
k
\end{array}\right) q^{k}(1-q)^{4-k} C_{k}
$$

packets successfully on the average. The intended packets constitute only one quarter of receptions.

Each node transmits $R(q)$ packets successfully on the average, since the number of successful receptions is always equal to the number of successful transmissions. This means that each link supports $R(q) / 4$ throughput. If the nodes use the routing protocol provided by Lemma 1 , it is seen that all $\lambda$ satisfying

$$
\frac{(N-1) \lambda \bar{L}}{4} \leq \frac{R(q)}{4}
$$

are uniformly achievable. Maximizing the right-hand side of (20) with respect to $q$ yields the achievability part of the theorem.

To see the converse, observe that the total traffic generated in the network must be less than the total throughput of the network, i.e.,

$$
N(N-1) \lambda \bar{L} \leq N R(q)
$$

for fixed $q$. This provides a bound on the maximum uniformly achievable rate regardless of what routing protocol is used. Optimization over $q$ gives the converse.

Omitting an additional $O(1 / N)$ factor, rewrite $\eta$ and $\eta_{\mathrm{ALOHA}}$ as

$$
\begin{aligned}
\eta & \simeq \frac{1}{\sqrt{N}} \max _{i=1, \ldots, 4} \frac{2 C_{i}}{i+1} \\
\eta_{\mathrm{ALOHA}} & \simeq \frac{1}{\sqrt{N}} \max _{0 \leq q \leq 1} \sum_{k=1}^{4}\left(\begin{array}{l}
4 \\
k
\end{array}\right) q^{k}(1-q)^{5-k} \frac{C_{k}}{2} .
\end{aligned}
$$

The preceding expressions show that the scaling law is $O(1 / \sqrt{N})$ and the per-node throughput goes to zero both with optimal scheduling and slotted ALOHA. This is similar to the capacity law observed in [1]. The main reason behind this fact is the uniform traffic which gives average path length $\bar{L}=O(\sqrt{N})$. Another factor affecting the capacity is the performance of the MAC protocol, which affects the coefficient but not the scaling law. As a numerical example, consider the MPR matrix for $M$-collision channel, $\boldsymbol{C}_{M}$. For $M \in\{1,2,3,4\}, \eta$, and $\eta_{\mathrm{ALOHA}}$ are given in Table II. It is seen that having the best MPR channel $C_{4}$ gives only 1.6 times improvement in $\eta$ over the conventional collision channel $C_{1}$. On the other hand, in the collision channel (first column in Table II), using optimal scheduling instead of slotted ALOHA provides about 6 times improvement.

\section{MANhatTAN Networks With FADING LinKS}

Suppose that each link in the Manhattan network is ON with probability $p$ and OFF with probability $1-p$. (Here, we mean undirected links; the links $(i, j)$ and $(j, i)$ are always in the same state.) Assume that the network policy does not know which links are ON or OFF, and the nodes transmit their packets without knowing if their link is ON or OFF. This will be called a network without LSI.

Suppose that node $i$ transmits to node $j$. If the link $(i, j)$ is $\mathrm{ON}$, and if $j$ is the only transmitter in $i$ 's neighborhood whose link with $i$ is ON, then the transmission is successful; it is unsuccessful otherwise. This channel can be expressed using the MPR matrix

$$
\boldsymbol{C}_{p}=\left(\begin{array}{ccccc}
1-p & p & & & \\
1-2 p(1-p) & 2 p(1-p) & 0 & & \\
1-3 p(1-p)^{2} & 3 p(1-p)^{2} & 0 & 0 & \\
1-4 p(1-p)^{3} & 4 p(1-p)^{3} & 0 & 0 & 0
\end{array}\right)
$$

Here, the entry $C_{k, 1}$ is the probability that $k$ neighbors transmit and one of them gets through, which is the case only if one link is ON and the rest $k-1$ are OFF; the probability of this event is $\left(\begin{array}{l}k \\ 1\end{array}\right) p(1-p)^{k-1}$.

For this channel, Theorem 4 gives the network capacity as

$$
\eta \simeq \frac{1}{\sqrt{N}} \max _{i=1, \ldots, 4} \frac{i(1-p)^{i-1}}{(i+1)} 2 p .
$$

Theorem 4 also gives a way to schedule packets optimally. The value of

$$
\begin{aligned}
\tau & =\underset{i=1, \ldots, 4}{\arg \max } \frac{i(1-p)^{i-1}}{(i+1)} \\
& = \begin{cases}1 & 1 \geq p \geq \frac{1}{4} \\
2 & \frac{1}{4} \geq p \geq \frac{1}{9} \\
3 & \frac{1}{9} \geq p \geq \frac{1}{16} \\
4 & \frac{1}{16} \geq p \geq 0\end{cases}
\end{aligned}
$$

determines which $\tau$-MPR pattern (Fig. 4) to use as a function of the severity of fading. From (21), it is apparent that one should use higher $\tau$ 's when $p$ is smaller. Using higher $\tau$ for small $p$ can be interpreted as multiuser diversity. For instance when $p$ is very small, in the neighborhood of the receiver there is a very small probability that there is more than a single-link ON. Therefore, 4-MPR scheduling (namely, "all neighbors transmit to the node in the center" strategy) does not lead to frequent collisions and increases the probability of successful transmission. The rates achievable with $\tau$-MPR scheduling, $\tau \in\{1,2,3,4\}$, are shown in Fig. 6.

It is an interesting question to ask what would be the improvement due to having and exploiting the LSI. The optimal policy again follows a similar idea in case of LSI: given the fading configuration, find and use the transmission schedule that maximizes the number of successful transmissions. However, it is very hard to compute the achievable rates for this case, since there are numerous fading configurations. The following theorem gives bounds on the capacity with LSI. 


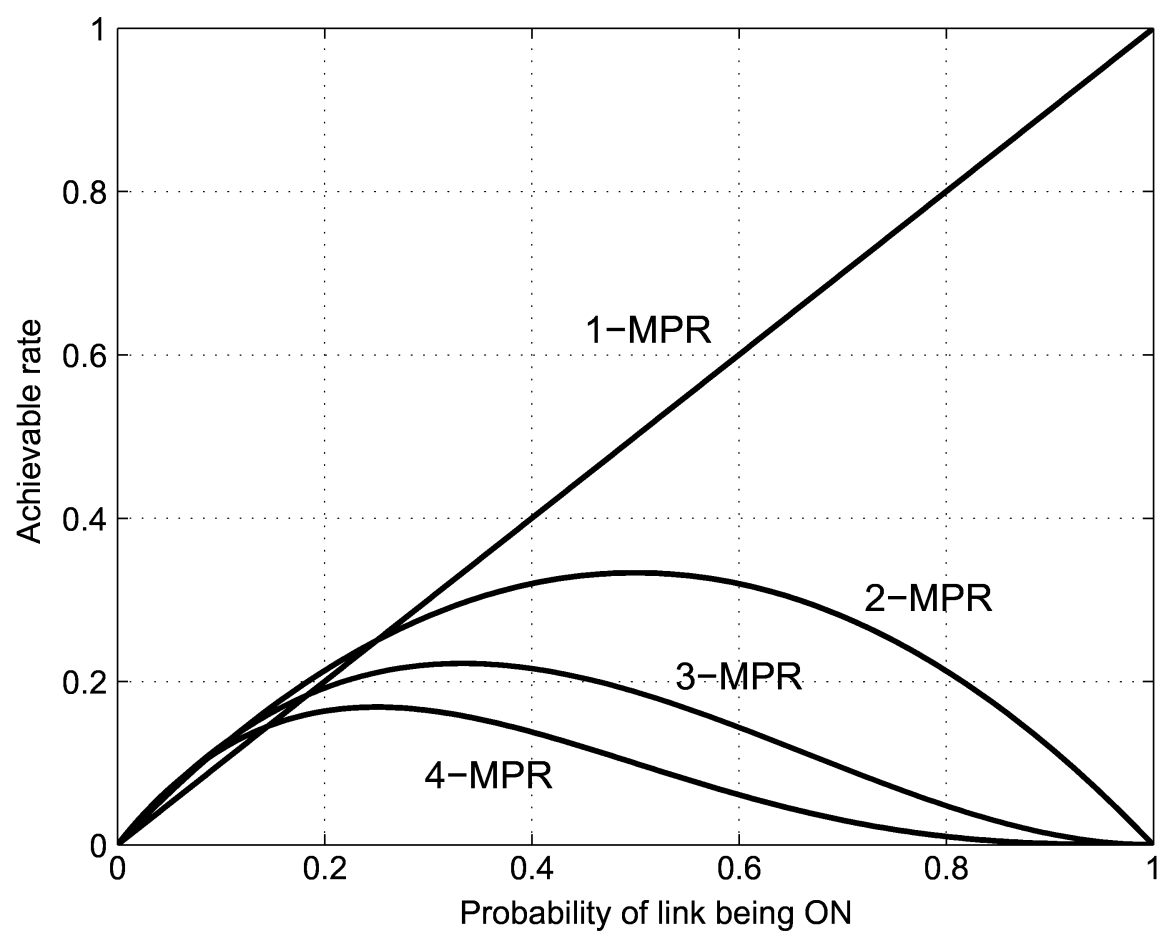

Fig. 6. $\left(\tau(1-p)^{\tau-1}\right) /(\tau+1) 2 p$ versus $p$. The upper envelope of the curves is $\eta \cdot \sqrt{N}$. When $p$ is small, the 4 -MPR gives the highest throughput and 1 -MPR gives the lowest.

Theorem 6: (Capacity of Manhattan Networks With LSI): Let $\eta^{\#}$ be the capacity of the Manhattan network with LSI. Then

$$
1 \leq \frac{\eta^{\#}}{\eta} \leq 2.86+O(1 / \sqrt{N})
$$

Moreover

$$
\lim _{\substack{N \rightarrow \infty \\ p \rightarrow 0}} \frac{\eta^{\#}}{\eta}=2.5 \quad \text { and } \quad \lim _{\substack{N \rightarrow \infty \\ p \rightarrow 1}} \frac{\eta^{\#}}{\eta}=1 .
$$

Proof: We will first discuss the extreme cases $p \simeq 0, p \simeq 1$. These two regimes are easier to understand, since there are simple strategies with performance close to the optimal.

If $p \simeq 0$, then very few links are $\mathrm{ON}$, and the optimal strategy is transmitting over almost every ON link. We describe a strategy that will be called all ons scheduled next. Let $a$ and $b$ be two nodes in the network (Fig. 7). In every slot, schedule a transmission over link $(a, b)$ if and only if the link $(a, b)$ is ON, and all of the other six links connecting $a$ and $b$ to their respective neighbors are OFF. Choose the direction of transmission randomly; $a$ to $b$ with probability $1 / 2$, and $b$ to $a$ with probability $1 / 2$. With this scheduling, the traffic that can be carried in each direction is $p(1-p)^{6} / 2$ (this is the probability that the link is scheduled in a direction). Using symmetric, shortest path routing, we see that rates below $2 p(1-p)^{6} /(N-1) \bar{L}$ are uniformly achievable. Furthermore, rates above $2 p /(N-1) \bar{L}$ are not uniformly achievable. This is true, since there are a total of $2 N$ undirected links in the network and the expected number of oN links is $2 p N$. Therefore, the transport capacity averaged over the link states is upper-bounded by $2 p N$. Theorem 2 can be extended to networks with multiple states by replacing the transport capacity by the average transport capacity (see Appendix A). Therefore, $\eta^{\#} \leq 2 p / \bar{L}$ follows.

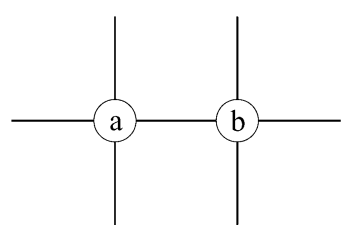

Fig. 7. Scheduling example with LSI.

We have just shown that

$$
\frac{2 p(1-p)^{6}}{\bar{L}} \leq \eta^{\#} \leq \frac{2 p}{\bar{L}} .
$$

When all sides are divided by

$$
\eta=\frac{4 p(1-p)^{3}}{5 \bar{L}}\left[1+O\left(\frac{1}{\sqrt{N}}\right)\right]
$$

we get

$$
\frac{5(1-p)^{3}}{2}\left[1+O\left(\frac{1}{\sqrt{N}}\right)\right] \leq \frac{\eta^{\#}}{\eta} \leq \frac{5}{2(1-p)^{3}}\left[1+O\left(\frac{1}{\sqrt{N}}\right)\right] .
$$

The left-hand side of (23) follows when we take the limit $p \rightarrow$ $0, N \rightarrow \infty$.

"All ONs scheduled" strategy almost achieves the capacity with LSI, which is $\eta^{\#} \simeq(2 p / \bar{L})$ for $p \simeq 0$. However, without LSI, the optimal strategy is 4-MPR scheduling which uses only $2 / 5$ of the available links (this fact can be seen by counting the number of used links in Fig. 4(d)). As a result, the "all ONs scheduled" strategy achieves $5 / 2$ times the throughput of 4-MPR scheduling.

Next, we will look at the regime $p \simeq 1$. Note that the capacity with LSI is always less than the capacity without fading, i.e., 


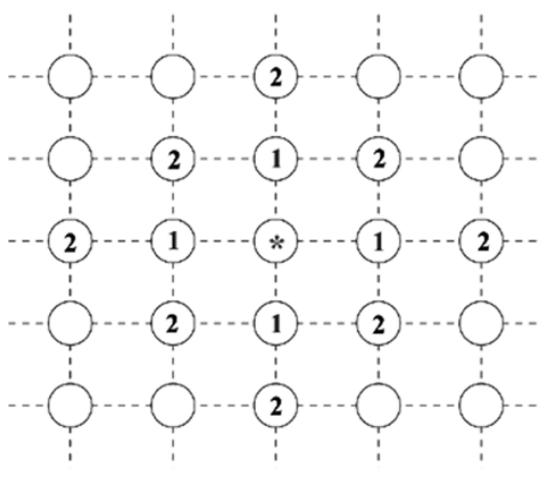

(a)

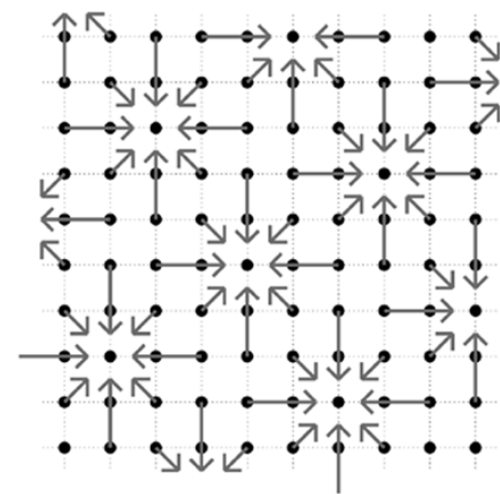

(b)

Fig. 8. (a) The neighbors of the two-hop connected Manhattan network are shown. Consider the node in the center (marked with $*$ ). It has total 12 neighbors, four of them are one-hop neighbors (marked with 1), and eight are two-hop neighbors (marked with 2). (b) 8-MPR scheduling in a Manhattan network with two-hop connectivity. The network is divided into groups of 13 nodes. In each group, the node in the center is the receiver, the receiver's two-hop neighbors are transmitters, and the one-hop neighbors of each receiver stay idle.

$\eta^{\#} \leq 1 / 2 \bar{L}$. Moreover, the capacity with LSI is greater than capacity without LSI, $\eta \leq \eta^{\#}$. Hence, the following holds:

$$
\frac{p}{2 \bar{L}}\left[1+O\left(\frac{1}{\sqrt{N}}\right)\right]=\eta \leq \eta^{\#} \leq \frac{1}{2 \bar{L}}
$$

for $p>1 / 4$. Divide all sides by $\eta$

$$
1 \leq \frac{\eta^{\#}}{\eta} \leq \frac{1}{p}\left[1+O\left(\frac{1}{\sqrt{N}}\right)\right] .
$$

Taking the limit $p \rightarrow 1, N \rightarrow \infty$ gives the right-hand side of (23). One conclusion is that if $p \simeq 1$ then almost all links are always ON, and with LSI using 1-MPR is almost optimal. The results for $p \simeq 0, p \simeq 1$ also suggest that the knowledge of LSI is more valuable when $p$ is small. For high values of $p$, LSI is less important; one can simply use 1-MPR scheduling.

Next, we will upper-bound the transport capacity for an arbitrary $p$. Let $\mathcal{E}_{\mathcal{L}}$ be the schedule maximizing the number of successful receptions for a given connectivity graph $(\mathcal{N}, \mathcal{L})$. Let $p(\mathcal{L})$ denote the probability of having graph $(\mathcal{N}, \mathcal{L})$ under the assumption of independent and identically distributed (i.i.d.) ON/OFF links. Without loss of generality, assume that each link $l$ in schedule $\mathcal{E}_{\mathcal{L}}$ is successful. Hence, the transport capacity averaged over the set of states is equal to

$$
\sum_{\mathcal{L}} p(\mathcal{L}) \sum_{l \in \mathcal{L}} 1\left(l \in \mathcal{E}_{\mathcal{L}}\right)
$$

Observe that $\sum_{l \in \mathcal{L}} 1\left(l \in \mathcal{E}_{\mathcal{L}}\right)$ is equal to

$$
\frac{1}{2} \sum_{i \in \mathcal{N}} 1\left((i, j) \in \mathcal{E}_{\mathcal{L}} \text { or }(j, i) \in \mathcal{E}_{\mathcal{L}} \text { for some } j\right)
$$

where the factor $1 / 2$ comes from the fact that each successfully transmitted packet is counted twice; once at the transmitter, once at the receiver. So, we can write the average transport capacity as

$$
\begin{aligned}
\sum_{\mathcal{L}} p(\mathcal{L}) & \frac{1}{2} \sum_{i \in \mathcal{N}} 1\left((i, j) \text { or }(j, i) \in \mathcal{E}_{\mathcal{L}} \text { for some } j\right) \\
& =\frac{1}{2} \sum_{i \in \mathcal{N}} \sum_{\mathcal{L}} p(\mathcal{L}) 1\left((i, j) \text { or }(j, i) \in \mathcal{E}_{\mathcal{L}} \text { for some } j\right) .
\end{aligned}
$$

The final sum $\sum_{\mathcal{L}} p(\mathcal{L}) 1\left((i, j)\right.$ or $(j, i) \in \mathcal{E}_{\mathcal{L}}$ for some $\left.j\right)$ is the expected number of successfully transmitted or received packets by node $i$. Since every node has four neighbors, this expectation is less than or equal to $1-(1-p)^{4}$, which is the probability that at least one out of four links is ON. Thus, we have proved the following upper bound:

$$
\begin{aligned}
\sum_{\mathcal{L}} p(\mathcal{L}) \sum_{l \in \mathcal{L}} 1\left(l \in \mathcal{E}_{\mathcal{L}}\right) & \leq \frac{1}{2} \sum_{i \in \mathcal{N}}\left(1-(1-p)^{4}\right) \\
& =\frac{N}{2}\left(1-(1-p)^{4}\right) .
\end{aligned}
$$

Since the average transport capacity divided by $\bar{L} N$ upperbounds the network capacity, we have $\eta^{\#} \leq\left(1-(1-p)^{4}\right) / 2 \bar{L}$. When we divide by $\eta$

$$
\begin{aligned}
\frac{\eta^{\#}}{\eta} & =\frac{\eta^{\#}}{\eta^{*}(1+O(1 / \sqrt{N}))} \\
& \leq\left[\max _{i=1, \ldots, 4} \frac{i(1-p)^{i-1}}{(i+1)}\right]^{-1} \frac{1-(1-p)^{4}}{2 p}+O\left(\frac{1}{\sqrt{N}}\right) .
\end{aligned}
$$

The last quantity is upper-bounded by $2.853 \cdots+O(1 / \sqrt{N})$, which is achieved at $p=0.1111 \ldots$. Therefore, (22) follows.

\section{OTHER REGULAR TOPOLOGIES AND OPTIMAL CONNECTIVITY}

Consider a Manhattan network with two-hop connectivity, i.e., every node is connected to neighbors two hops or one hop away (there are 12 such neighbors, see Fig. 8(a)). Consider the scheduling pattern in Fig. 8(b). This pattern can be used under the assumption that the nodes can perfectly receive eight packets simultaneously (i.e., the MPR matrix is $C_{8}$-this channel can be viewed as an abstraction for CDMA with high spreading gain). When we tile the network with such a pattern, approximately $8 / 13$ of the nodes are transmitters and the $1 / 13$ nodes are receivers. Each transmitted packet moves two distance units, and the expected progress (rate-distance product) with this scheduling is

$$
\frac{16}{13} N+O(\sqrt{N})
$$

This quantity is higher than the expected progress with the 4-MPR pattern in a one-hop connected network (i.e., the network considered in previous sections). In 4-MPR scheduling, approximately $1 / 5$ of the nodes are scheduled as receivers and 


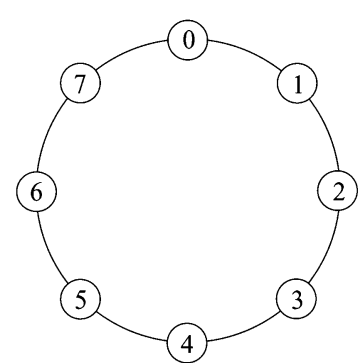

(a)

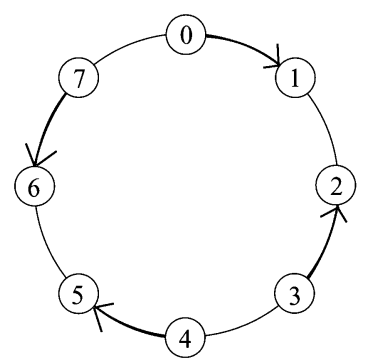

(b)

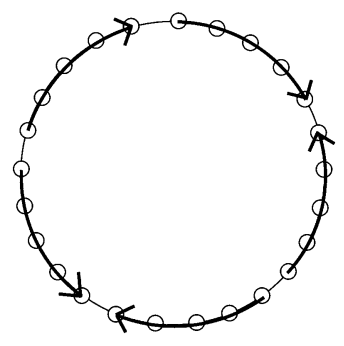

(c)

Fig. 9. (a) A ring network with eight nodes. The circumference is of unit length, and the distance between any two neighbors is $1 / 8$ units. (b) 1 -RING scheduling in a ring with eight nodes. (c) 4-RING scheduling in a ring with 25 nodes.

each receiver gets four packets moving a single distance unit. Hence, the expected progress with 4-MPR scheduling is

$$
\frac{4}{5} N+O(\sqrt{N}) .
$$

Here, we see that multipacket receptions, which is the case in CDMA, cannot be exploited sufficiently if the connectivity is not high enough. In the next theorem, we argue that the increase in expected progress translates to increased achievable rates.

Theorem 7: (Capacity of the Two-Hop Connected Network):

Let $\eta_{2-\mathrm{HOP}}$ be the capacity of a two-hop connected Manhattan network with MPR matrix $\boldsymbol{C}_{8}$. Then

$$
\frac{16}{13} \frac{1}{\bar{L}}+O\left(\frac{1}{N}\right) \leq \eta_{2-\mathrm{HOP}} \leq \frac{16}{9} \frac{1}{\bar{L}} .
$$

The lower bound is about $54 \%$ higher than

$$
\eta=(4 / 5)(1 / \bar{L})+O(1 / N)
$$

the capacity of a one-hop connected Manhattan network with $\boldsymbol{C}_{8}{ }^{3}$

Proof: The achievability of the lower bound is essentially the same as the achievability of $\eta$ in one-hop connected Manhattan networks. The differences and similarities are the following. In a two-hop connected network, it is advantageous for packets to move two hops per transmission rather than one hop. The routing protocol achieving the lower bound uses this idea; packets keep jumping two hops until they reach to the destination, or to a one-hop neighbor of the destination after which they have a single hop to go. The traffic load over links can be balanced in a way similar to Lemma 1. Moreover, in a large network, the traffic for the one-hop neighbors is negligible compared to traffic for the two-hop neighbors. In a load-balanced network, the 8-MPR pattern and its shifted versions achieve the lower bound.

Next, we will prove the upper bound. Consider a transmission schedule $\mathcal{E}$. Let $k$ be a node hearing $j_{r}$ transmissions from its $r$-hop neighbors, $r=1,2$, intended for itself. Let $A_{j_{1}, j_{2}}$ be the number of all such nodes in $\mathcal{E}$. Observe that $j_{1}, j_{2}$ must lie in

$$
\mathcal{J}=\left\{\left(j_{1}, j_{2}\right) \in \mathbb{Z}_{+}^{2}: j_{1} \leq 4, j_{1}+j_{2} \leq 8\right\}
$$

(this is under the assumption that every transmitter is successful; otherwise, we can eliminate some unnecessary transmissions

\footnotetext{
${ }^{3}$ The lower bound is achievable for every $\boldsymbol{C}_{M}, M \geq 8$.
}

from $\mathcal{E}$ without affecting the successful ones). $A_{j_{1}, j_{2}}$ must satisfy

$$
\sum_{\left(j_{1}, j_{2}\right) \in \mathcal{J}} A_{j_{1}, j_{2}}\left(1+j_{1}+j_{2}\right) \leq N
$$

since the total number of nodes is less than $N$. Further, observe that

$$
\frac{1}{\bar{L} N} \sum_{l \in \mathcal{L}} d(l) \Pi(l ; \mathcal{E})=\frac{1}{\bar{L} N} \sum_{\left(j_{1}, j_{2}\right) \in \mathcal{J}} A_{j_{1}, j_{2}}\left(j_{1}+2 j_{2}\right) .
$$

Maximizing (27) under the constraint (26), and $A_{i j}$ being nonnegative and real gives

$$
\begin{aligned}
\frac{1}{\bar{L} N} \sum_{l \in \mathcal{L}} d(l) \Pi(l ; \mathcal{E}) & \leq \frac{1}{\bar{L} N} \max _{\left(j_{1}, j_{2}\right) \in \mathcal{J}}\left\{N \frac{j_{1}+2 j_{2}}{1+j_{1}+j_{2}}\right\} \\
& =\frac{16}{9} \frac{1}{\bar{L}} .
\end{aligned}
$$

We get the upper bound by invoking Theorem 2 .

The previous theorem bounds the capacity for a given MPR matrix. Namely, it addresses the question "Given the physical layer, what is the best MAC/routing/connectivity?" For example, this question is relevant to CDMA networks, where multipacket reception already exists. To find the ultimate network capacity, one ought to optimize with respect to the physical layer as well. However, we do not attempt this optimization in this paper.

Next, we will consider ring networks. Assume that the nodes are uniformly placed on a ring with unit circumference (see Fig. 9). The distance between node $i$ and node $j$ is given by $d(i, j)=\min \{\delta, 1-\delta\}$, where $\delta=|i-j| / N$. We will consider the following simple reception model which is an extension of the collision channel: If $\left\{\left(i_{k}, j_{k}\right): k=1,2, \ldots, K\right\}$ is a set of transmitter-receiver pairs then the transmission from $i_{k}$ to $j_{k}$ is successful if 4

$$
d\left(i_{r}, j_{k}\right)>d\left(i_{r}, j_{r}\right), \quad \text { for all } r \neq k .
$$

The rationale behind this model is that each transmitter $i_{r}$ uses just sufficient power to reach its destination $j_{r}$; this transmission causes interference to all nodes within $d\left(i_{r}, j_{r}\right)$ neighborhood of the transmitter, and negligible interference to the nodes outside. Transmission from $i_{k}$ to $j_{k}$ is successful only if $j_{k}$ is sufficiently far apart from every other transmitter.

\footnotetext{
${ }^{4}$ The results in this section can be generalized with minor changes to the model $d\left(i_{r}, j_{k}\right)>(1+\Delta) d\left(i_{r}, j_{r}\right)$ for some $\Delta>0$.
} 
Lemma 2: Suppose that each transmission $(i, j)$ is represented with an arrow from $i$ to $j$ with length $d(i, j)$. Then, the arrows corresponding to two successful transmissions do not intersect.

Proof: The proof is by contradiction. Consider two successful transmissions $\left(i_{1}, j_{1}\right),\left(i_{2}, j_{2}\right)$. If $j_{2}$ were between $i_{1}$ and $j_{1}$, then $\left(i_{2}, j_{2}\right)$ could not be successful. Similarly, if $i_{2}$ were between $i_{1}$ and $j_{1}$, and $j_{2}$ were outside, then the $\left(i_{2}, j_{2}\right)$ arrow would either go over $j_{1}$ (which makes $\left(i_{1}, j_{1}\right)$ unsuccessful), or would go over $i_{1}$. In the latter case, to make $\left(i_{2}, j_{2}\right)$ successful $j_{2}$ must be at least $d\left(i_{1}, j_{1}\right)$ apart from $i_{1}$. However, if $j_{2}$ is placed that far apart, then the interference from $i_{2}$ makes $\left(i_{1}, j_{1}\right)$ unsuccessful.

Remark: The total lengths of the arrows cannot exceed the circumference of the ring. Therefore, Lemma 2 implies that the transport capacity of the ring is upper-bounded by 1 .

Next, we will see how the transport capacity of the ring can be approached. Two cases will be considered: one-hop connected ring (nodes are restricted to communicate with their nearest two neighbors) and the ring without constraint. An important observation is that in the one-hop connected ring we can use a one-dimensional analogue of 1-MPR scheduling; see the so-called 1-RING scheduling in Fig. 9(b). The 1-RING pattern can be used to cover all ring, and it uses half of the space available. That is, when the number of nodes is even, half of the nodes get a chance to transmit and the distance of each transmission is $1 / N$. Therefore, the expected progress (rate-distance product) is $1 / 2$. Similarly, the expected progress of 1 -RING scheduling for odd $N$ can be obtained as $1 / 2+O(1 / N)$.

In the ring without constraint there are other possibilities for communication. In particular, consider the so-called $\tau-R I N G$ scheduling in which every packet jumps $\tau$ hops (see the 4-RING example in Fig. 9(c)). In $\tau$-RING, the transmitter-receiver pairs are given by

$$
\begin{aligned}
&(0, \tau),(2 \tau+1, \tau+1),(2(\tau+1), 2(\tau+1)+\tau), \\
&(3(\tau+1)+\tau, 3(\tau+1)), \ldots
\end{aligned}
$$

where the list is truncated at the point where either a transmitter or a receiver index goes above $N-1$. If the $\tau$-RING pattern perfectly covers the network (i.e., $N$ is divisible by $\tau+1$ ), then $N /(\tau+1)$ transmissions, each over $\tau$-hops, are scheduled. This means the expected progress of $\tau$-RING is $\tau /(\tau+1)$. For general $N, \tau$-RING may not cover the network, but the expected progress is $\tau /(\tau+1)+O(1 / N)$.

From these observations, we see that the expected progress is an increasing function of $\tau$, and the 1-RING scheduling gives the minimum expected progress. In the limit $\tau \rightarrow \infty$, we get $\tau /(\tau+1) \rightarrow 1$. Since the transport capacity cannot be larger than 1 , we see that the $\tau$-RING patterns achieve the transport capacity in the limit. The following theorem gives analogous results for the network capacity.

Theorem 8: (Capacity of the Ring): The capacity of one-hop connected ring is

$$
\eta_{1-\mathrm{RING}}=\frac{2}{N}+O\left(\frac{1}{N^{2}}\right)
$$

On the other hand, the capacity of the unconstrained ring satisfies

$$
\frac{4 \tau}{\tau+1} \frac{1}{N}+O\left(\frac{1}{N^{2}}\right) \leq \eta_{\mathrm{RING}} \leq \frac{4}{N}
$$

for every $\tau=1,2, \ldots$. Therefore, the capacity of the unconstrained ring is double that of the minimally connected ring.

Proof: 1-RING scheduling and its shifted versions achieve (28). The general $\tau$-RING, $\tau \geq 1$ achieves the lower bound in (29). The routing uses shortest paths; packets hop $\tau$ hops until they reach the $\tau$ neighborhood of their destination. The shortest path routing, as before, balances the traffic. Further, for large $N$, it can be seen that only a negligible fraction of the traffic is diverted to neighbors less than $\tau$ hops away (for such packets $\tau^{\prime}<\tau$ RING scheduling is used). The main ideas are the same as the ones in Theorems 4 and 7 , and we will not go through the details to avoid repetition.

The average path length in the ring satisfies $\bar{L} \geq 1 / 4$. Furthermore

$$
\bar{L}=1 / 4+O(1 / N) .
$$

We will do this computation only for odd $N$; the other case is almost identical.

$$
\begin{aligned}
\bar{L} & =\frac{1}{N-1} \sum_{i=1}^{N-1} d(0, i) \\
& =\frac{1}{(N-1)} \sum_{i=-(N-1) / 2}^{(N-1) / 2} \frac{|i|}{N} \\
& =\frac{2}{N(N-1)} \sum_{i=0}^{(N-1) / 2} i \\
& =\frac{2}{N(N-1)} \frac{N^{2}-1}{8} \\
& =\frac{N+1}{4 N} \\
& =\frac{1}{4}+O\left(\frac{1}{N}\right) .
\end{aligned}
$$

In the unconstrained ring, the transport capacity is upperbounded by 1 as argued after Lemma 2 . Therefore, the capacity is $\leq 1 /(N \bar{L})$ by Theorem 2 . Since $\bar{L} \geq 1 / 4$, we get the upper bound $\eta_{\mathrm{RING}} \leq 4 / N$.

In the minimally connected ring, we need to prove that the transport capacity is upper-bounded by $1 / 2+O(1 / N)$ under the restriction that nodes can only talk to immediate neighbors; this can be easily done using a technique identical to the ones in Theorems 4 and 7 . The upper bound for $\eta_{1-\text { RING }}$ follows.

\section{CONCLUSION}

In this paper, we first characterized the capacity region of arbitrary networks, and provided general scheduling policies that achieve every rate inside the capacity region. We then gave an upper bound on the network capacity in terms of the transport capacity, and argued that the stability region of the network is identical to capacity region under a mild assumption on the reception channel. We then obtained a closed-form expression for 
the capacity of Manhattan networks and analyzed the impact of link fading, link state information and the topology information on achievable rates. We also compared a suboptimal scheme that uses ALOHA as its medium access to the optimal policy that jointly optimizes medium access and routing. We finally examined the effect of nonminimal connectivity on the capacity of Manhattan and ring networks.

The results for regular networks have ramifications for MAC in arbitrary networks. In Manhattan networks with multipacket receiving nodes, the $\tau$-MPR patterns (Figs. 4 and 8(b)), namely "neighboring nodes transmit into the center" strategy, was shown to be optimal for medium access. The $\tau$-MPR scheduling locally resembles an uplink especially for $\tau$ large. We expect this type of scheduling to be useful in arbitrary networks where multipacket reception is possible with multiple receive antennas or spread spectrum. The 1-MPR scheduling and $\tau$-RING scheduling (Figs. 4 and 9), namely, "transmitters turn each other their back and transmit" strategy, were shown to be optimal in networks without multipacket reception. We expect this idea to be useful in wireless networks with parts locally resembling a one-dimensional topology. Examples include a wireless local-area network (LAN) in a corridor, or a group of nodes on a street or a highway.

There is an important open question related to the capacity of arbitrary networks: Can the transport capacity upper bound be used for determining the capacity of arbitrary networks? In regular networks it is shown that the transport capacity provides tight upper bounds on the capacity. This upper bound is not always achievable in arbitrary networks, however, it suggests a general duality relation between the transport capacity and network capacity. To see what we mean by duality, recall that the transport capacity upper bound, inequality (8), is valid for all distance metrics satisfying the triangle inequality. Hence, the upper bound minimized over all distance metrics is still an upper bound. But, how does the minimized upper bound compare with the network capacity? Under what conditions is the minimized upper bound achievable ${ }^{5}$ These are some questions which seem to deserve further attention.

\section{APPENDIX}

\section{A. Extensions to a General Network Model With Asymmetric and Time-Varying Reception Probabilities}

In this appendix, we provide extensions of the capacity and stability results in Section III for the following more general network model.

i) Let $\mathcal{V}$ be the set of possible network states. Assume that the network state $v \in \mathcal{V}$ changes from slot to slot according to a stationary and ergodic stochastic process. Scheduling policies are assumed to have instantaneous knowledge of the network state while making transmission decisions.

\footnotetext{
${ }^{5}$ We are grateful to an anonymous reviewer who pointed out that for a given distance metric the upper bound is achievable if only if the traffic requirements is such that the network can continuously operate according to the schedule(s) that attain the maximum in (8).
}

ii) The reception probabilities are specified by a conditional probability density function (pdf) $\pi(\cdot ; \mathcal{E}, v)$, where $v \in \mathcal{V}$ is the state and $\mathcal{E}$ is the set of transmissions. Here, $\pi(\mathcal{F} ; \mathcal{E}, v)$ is the probability that the set of successful receptions is $\mathcal{F} \subset \mathcal{E}$ given that the network is in state $v$ and the set of transmitters is $\mathcal{E}$. We have the property $\sum_{\mathcal{F} \subset \mathcal{E}} \pi(\mathcal{F} ; \mathcal{E}, v)=1$ for all $\mathcal{E}, v$.

iii) Define the set $\mathcal{L}=\{(i, j): i \neq j, i, j \in \mathcal{N}\}$. Let $\mathrm{E}$ be the all possible subsets (i.e., the power set) of $\mathcal{L}$. In this general model, the topological properties of the network and transmission constraints are not described by a connectivity graph, but they are embedded into the pdf $\pi$. That is, if a set of transmissions $\mathcal{E} \in \mathrm{E}$ is physically impossible in state $v$, then the set of successful receptions is empty with probability one.

The strength of this model is its conciseness and generality. As in information theory, all channel properties are specified by a set of conditional pdfs $\{\pi(\cdot ; \mathcal{E}, v)\}_{\mathcal{E}, v}$. By substituting specific $\pi$, the MPR model and several other models can be obtained as special cases [23], [24], [30], [39], [40].

The definition of RTD policies can be generalized to this model by considering a set of conditional pdfs $\{p(\mathcal{E} ; v)\}_{\mathcal{E}}$, one for each state $v$. If the network is in state $v$, a random schedule $\mathcal{E}$ is chosen according to the pdf $\{p(\mathcal{E} ; v)\}_{\mathcal{E}}$. The routing and queuing disciplines are as before.

Let notation

$$
\Pi(\mathcal{F} ; \mathcal{E}, v)=\sum_{\mathcal{F}^{\prime}: \mathcal{F} \subset \mathcal{F}^{\prime}} \pi\left(\mathcal{F}^{\prime} ; \mathcal{E}, v\right)
$$

denote the marginal probability of success for the set $\mathcal{F}$. The following theorem generalizes Theorems 1-3.

\section{Theorem 9:}

i) A rate vector $\left(\lambda_{i j}: i, j \in \mathcal{N}\right)$ is achieved by an RTD policy if

$$
\sum_{i, j \in \mathcal{N}} \sum_{P \in \mathrm{P}_{i j}: l \in P} x_{P} \lambda_{i j} \leq \mathbb{E}_{v}\left[\sum_{\mathcal{E} \in \mathrm{E}} \Pi(l ; \mathcal{E} ; v) p(\mathcal{E} ; v)\right]
$$

for all $l \in \mathcal{L}$. The capacity region is equal to the union of all $\left(\lambda_{i j}: i, j \in \mathcal{N}\right)$ achievable with some RTD policy.

ii) The network capacity satisfies

$$
\eta \leq \frac{1}{\bar{L} N} \mathbb{E}_{v}\left[\max _{\mathcal{E} \in \mathrm{E}} \sum_{l \in \mathcal{L}} d(l) \Pi(l ; \mathcal{E}, v)\right]
$$

where the right-hand side is the transport capacity averaged over different states.

iii) The stability and capacity regions are identical if

$$
\Pi\left(\mathcal{F} ; \mathcal{E}^{\prime}, v\right) \leq \Pi(\mathcal{F} ; \mathcal{E}, v)
$$

holds for every $\mathcal{F} \subset \mathcal{E} \subset \mathcal{E}^{\prime}, v \in \mathcal{V}$. If an RTD policy satisfies (30) with strict inequalities for some $\left(\lambda_{i j}\right.$ : $i, j \in \mathcal{N})$, then the network with arrival rate $\left(\lambda_{i j}: i, j \in\right.$ $\mathcal{N}$ ) is stabilized by the same RTD policy.

Proof: See [28]. 


\section{B. Proof of Proposition 2}

The average distance a packet originating from node $i$ travels is the same for all $i$. As a result of this symmetry, we can compute $\bar{L}$ by averaging the distances between the node $(0,0)$ and the other nodes in the network

$$
\begin{aligned}
\bar{L}= & \frac{1}{N-1} \sum_{x=0}^{\sqrt{N}-1} \sum_{y=0}^{\sqrt{N}-1} d\{(0,0),(x, y)\} \\
= & \frac{1}{N-1} \sum_{x=0}^{\sqrt{N}-1} \sum_{y=0}^{\sqrt{N}-1} \min \{x, \sqrt{N}-x\} \\
& +\min \{y, \sqrt{N}-y\} \\
= & \frac{2 \sqrt{N}}{N-1} \sum_{x=1}^{\sqrt{N}-1} \min \{x, \sqrt{N}-x\} .
\end{aligned}
$$

The two cases follow from the last expression.

\section{Proof of Lemma 1}

For a given source-destination pair, there exist many routes with the minimum path length. It is the objective of routing to use the shortest distances while distributing the load uniformly. In this part of the appendix, we show that all $\left\{x_{P}\right\}$ that specify a symmetric and shortest path routing protocol satisfy

$$
\sum_{i, j \in \mathcal{N}} \sum_{P \in \mathrm{P}_{i j}: l \in P} x_{P}=\frac{(N-1) \bar{L}}{4}, \quad \forall l \in \mathcal{L}
$$

which suffices to prove the lemma. The following notations will be used.

i) Let $|P|$ denote to number of links on a path $P$. Routing probabilities $\left\{x_{P}\right\}$ specify a shortest path routing protocol if $x_{P}>0, P \in \mathrm{P}_{i j}$ implies $|P|=d(i, j)$.

ii) Let $\mathbb{Z}^{2}$ be the set of all $(x, y)$ with integer coordinates. Let $(x, y) \in \mathbb{Z}^{2}$ refer to the node with coordinates $(\bmod (x), \bmod (y))$ where $\bmod (x)=x \bmod \sqrt{N}$ is the usual modulo function. For $i, \delta \in \mathbb{Z}^{2}$, let $i+\delta$ denote the $\delta$ translation of node $i$. Similarly, $l+\delta, P+\delta$ refer to the $\delta$ translations of link $l$ and path $P$, respectively. We call the routing probabilities $\left\{x_{P}\right\}$ translation invariant if

$$
x_{P}=x_{P+\delta}
$$

is satisfied for all $i, j \in \mathcal{N}, P \in \mathrm{P}_{i j}, \delta \in \mathbb{Z}^{2}$.

iii) Let the vertical reflection of node $i=(x, y)$ be $i_{\uparrow}=$ $(x,-y)$, the horizontal reflection be $i_{\leftrightarrow}=(-x, y)$, and the rotation be $i_{\circlearrowleft}=(-y, x)$. Reflections and rotations of links and paths are defined similarly, and are denoted by $l_{*}, P_{*}$ where $* \in\{\uparrow, \leftrightarrow, \circlearrowleft\}$. Let 0 denote the node at the coordinate $(0,0)$, i.e., origin node. We call translation-invariant routing probabilities $\left\{x_{P}\right\}$ symmetric if for all $P \in \mathrm{P}_{0 j}$

$$
x_{P}=x_{P_{\uparrow}}=x_{P_{\leftrightarrow}}=x_{P_{\circlearrowleft}} .
$$

Example: Consider a source-destination pair $i, j \in \mathcal{N}$. Let

$$
\mathrm{P}_{i j}^{\prime}=\left\{P \in \mathrm{P}_{i j}:|P|=d(i, j)\right\}
$$

and

$$
x_{P}= \begin{cases}\frac{1}{\left|\mathrm{P}_{i j}^{\prime}\right|}, & \text { if } P \in \mathrm{P}_{i j}^{\prime} \\ 0, & \text { otherwise. }\end{cases}
$$

The probabilities $\left\{x_{P}\right\}$ specify a symmetric, shortest path routing protocol.

Denote the origin node with 0 . Next, we will argue that if the $\left\{x_{P}\right\}$ is translation invariant then for every link $l$

$$
\sum_{i, j \in \mathcal{N}} \sum_{P \in \mathrm{P}_{i j}: l \in P} x_{P}=\sum_{j \in \mathcal{N}} \sum_{P \in \mathrm{P}_{0 j}} x_{P} \psi(P, l)
$$

where

$$
\psi(P, l)=\sum_{i \in \mathcal{N}} 1(l \in P+i) .
$$

The links in a Manhattan network are in four directions: up, down, left, right. The number $\psi(P, l)$ is the number of links in $P$ which are in the same direction with $l$. Definition (35) assures that $\psi(P, l)$ depends on the direction of $l$ but not on its location, i.e., $\psi(P, l)=\psi(P, l+\delta)$ for every $\delta$.

Equation (34) can be interpreted as follows. Suppose that the network traffic is uniform and equal to 1 for every source-destination pair. The left-hand side of (34) is the routing load over link $l$. Equation (34) implies that the routing load on link $l$ depends on the direction of $l$, but not on its location (this is due to translation invariance). Moreover, (34) suggests an alternative way of computing the routing load over link $l$ : Fix the origin as the source node, and add up the traffic from the origin to the other nodes, passing through links in the same direction with $l$.

To see (34), make a change of variables $j^{\prime}=j-i$

$$
\begin{aligned}
& \sum_{i, j \in \mathcal{N}} \sum_{P \in \mathrm{P}_{i j}} x_{P} 1(l \in P) \\
& =\sum_{j^{\prime} \in \mathcal{N}} \sum_{i \in \mathcal{N}} \sum_{P \in \mathrm{P}_{i, j^{\prime}+i}} x_{P} 1(l \in P) \\
& =\sum_{j^{\prime} \in \mathcal{N}} \sum_{P \in \mathrm{P}_{0, j^{\prime}}} \sum_{i \in \mathcal{N}} x_{P+i} 1(l \in P+i) \\
& =\sum_{j^{\prime} \in \mathcal{N}} \sum_{P \in \mathrm{P}_{0, j^{\prime}}} x_{P} \sum_{i \in \mathcal{N}} 1(l \in P+i) .
\end{aligned}
$$

The last equality is due to the translation invariance of the routing.

From the definition of $\psi$, it follows that for all $* \in\{\uparrow, \leftrightarrow, \circlearrowleft\}$

$$
\psi(P, l)=\psi\left(P_{*}, l_{*}\right)
$$

Therefore, if the routing is symmetric then

$$
\begin{aligned}
\sum_{j \in \mathcal{N}} \sum_{P \in \mathrm{P}_{0 j}} x_{P} \psi(P, l) & =\sum_{j \in \mathcal{N}} \sum_{P \in \mathrm{P}_{0 j}} x_{P_{*}} \psi\left(P_{*}, l_{*}\right) \\
& =\sum_{j \in \mathcal{N}} \sum_{P \in \mathrm{P}_{0 j}} x_{P} \psi\left(P, l_{*}\right) .
\end{aligned}
$$

The first equality follows from (33) and (36). The second equality is due to the fact that if we map every $P \in \mathrm{P}_{0 j}$ to $P_{*}$ then we again obtain the set $\mathrm{P}_{0 j}$. 
Let $l$ be a link pointing up. Then, $l_{\circlearrowleft}, l_{\uparrow},\left(l_{\circlearrowleft}\right)_{\leftrightarrow}$ are vectors pointing left, down, and right, respectively. If the routing is symmetric, then (34) and (37) ensure that the traffic on links $l, l_{\circlearrowleft}, l_{\uparrow},\left(l_{\circlearrowleft}\right)_{\leftrightarrow}$ are the same.

Let the routing probabilities $\left\{x_{P}\right\}$ specify a symmetric, shortest path routing protocol. Next, we will show that $\left\{x_{P}\right\}$ satisfies (32). It follows from definitions that

$$
|P|=\psi(P, l)+\psi\left(P, l_{\circlearrowleft}\right)+\psi\left(P, l_{\uparrow}\right)+\psi\left(P,\left(l_{\circlearrowleft}\right)_{\leftrightarrow}\right) .
$$

Therefore,

$$
\begin{aligned}
\sum_{i, j \in \mathcal{N}} \sum_{P \in \mathrm{P}_{i j}: l \in P} x_{P}= & \sum_{j \in \mathcal{N}} \sum_{P \in \mathrm{P}_{0 j}} x_{P} \psi(P, l) \\
= & \frac{1}{4} \sum_{j \in \mathcal{N}} \sum_{P \in \mathrm{P}_{0 j}} x_{P}\left(\psi(P, l)+\psi\left(P, l_{\circlearrowleft}\right)\right. \\
& \left.+\psi\left(P, l_{\uparrow}\right)+\psi\left(P,\left(l_{\circlearrowleft}\right)_{\leftrightarrow}\right)\right) \\
= & \frac{1}{4} \sum_{j \in \mathcal{N}} \sum_{P \in \mathrm{P}_{0 j}} x_{P}|P| \\
= & \frac{1}{4} \sum_{j \in \mathcal{N}} d(0, j) \\
= & \frac{(N-1) \bar{L}}{4} .
\end{aligned}
$$

The first equality is due to (34). The second in due to (37). The third one is due to (38). The fourth one is because $|P|=d(0, j)$ for each $P \in \mathrm{P}_{o j}$ and $\sum_{P \in \mathrm{P}_{0 j}} x_{P}=1$. The last equality follows from the definition of $\bar{L}$ and the symmetry of the network topology.

\section{ACKNOWLEDGMENT}

The authors would like to thank the anonymous reviewers for their careful reading and extensive comments. The first author acknowledges helpful discussions with S. Adireddy, P. C. Budianu, and V. Naware. We would like to thank V. Naware also for proofreading the final manuscript.

\section{REFERENCES}

[1] P. Gupta and P. R. Kumar, "The capacity of wireless networks," IEEE Trans. Inf. Theory, vol. 46, no. 2, pp. 388-404, Mar. 2000.

[2] M. Grossglauser and D. N. C. Tse, "Mobility increases the capacity of wireless adhoc networks," IEEE/ACM Trans. Networking, vol. 10, no. 4, pp. 477-486, Aug. 2002.

[3] S. Ghez, S. Verdú, and S. Schwartz, "Stability properties of slotted Aloha with multipacket reception capability," IEEE Trans. Autom. Control, vol. 33, no. 7, pp. 640-649, Jul. 1988.

[4] D. Bertsekas and R. Gallager, Data Networks. Englewood Cliffs, NJ: Prentice Hall, 1992.

[5] J. Silvester and L. Kleinrock, "On the capacity of multihop slotted ALOHA networks with regular structure," IEEE Trans. Commun., vol. COM-31, no. 8, pp. 974-982, Aug. 1983.

[6] J. A. Silvester, "On the spatial capacity of packet radio networks," Ph.D. dissertation, Univ. Calif., Los Angeles, May 1980.

[7] E. Arikan, "Some complexity results about packet radio networks," IEEE Trans. Inf. Theory, vol. IT-30, no. 4, pp. 681-685, Jul. 1984.
[8] B. E. Hajek and G. Sasaki, "Link scheduling in polynomial time," IEEE Trans. Inf. Theory, vol. 34, no. 6, pp. 910-917, Sep. 1988.

[9] S. Toumpis and A. J. Goldsmith, "Capacity regions for wireless ad hoc networks," IEEE Trans. Wireless Commun., vol. 2, no. 4, pp. 736-748, Jul. 2003.

[10] _ - "Performance, optimization, and cross-layer design of media access protocols for wireless ad hoc networks," in Proc. Int. Conf. Communications (ICC), Anchorage, AK, May 2003, pp. 2234-2240.

[11] J. Li, C. Blake, D. D. Couto, H. Lee, and R. Morris, "Capacity of ad hoc wireless networks," in Proc. 7th ACM Int. Conf. Mobile Computing and Networking (Mobicom 01), Rome, Italy, Jul. 2001, pp. 61-69.

[12] M. Gastpar and M. Vetterli, "On the capacity of wireless networks: The relay case," in Proc. IEEE INFOCOM'02, vol. 3, New York, Jun. 2002, pp. $1577-1586$.

[13] P. Gupta and P. R. Kumar, "Toward an information theory of large networks: An achievable rate region," IEEE Trans. Inf. Theory, vol. 49, no. 8, pp. 1877-1894, Aug. 2003.

[14] L.-L. Xie and P. R. Kumar, "A network information theory for wireless communication: Scaling laws and optimal operation," IEEE Trans. Inf. Theory, vol. 50, no. 5, pp. 748-767, May 2004.

[15] S. R. Kulkarni and P. Viswanath, "A deterministic approach to throughput scaling in wireless networks," IEEE Trans. Inf. Theory, vol. 50, no. 6, pp. 1041-1049, Jun. 2004.

[16] O. Arpacioglu and Z. J. Haas, "On the scalability and capacity of wireless networks with omnidirectional antennas," in Proc. Information Processing in Sensor Networks (IPSN'04), Berkeley, CA, Apr. 2004, pp. 169-177.

[17] M. Franceschetti, O. Dousse, D. Tse, and P. Thiran, "Closing the gap in the capacity of random wireless networks," in Proc. IEEE Int Symp. Information Theory (ISIT'04), Chicago, IL, Jun./Jul. 2004, p. 438.

[18] L. Kleinrock and J. Silvester, "Spatial reuse in multihop packet radio networks," Proc. IEEE, vol. 75, no. 1, pp. 156-167, Jan. 1987.

[19] L. Tong, Q. Zhao, and G. Mergen, "Multipacket reception in random access wireless networks: From signal processing to optimal medium access control," IEEE Commun. Mag. (Special Issue on Design Methodologies for Adaptive and Multimedia Networks), vol. 39, no. 11, pp. 108-112, Nov. 2001.

[20] E. Modiano, "A dynamic adaptive multi-receiver random access protocol for the code division multiple access channel," in Proc. Conf. Personal, Indoor, and Mobile Radio Communications (PIMRC'95), vol. 2, Toronto, ON, Canada, Sep. 1995, pp. 799-803.

[21] Q. Zhao and L. Tong, "A dynamic queue protocol for multiaccess wireless networks with multipacket reception," IEEE Trans. Wireless Commun., to be published.

[22] S. Adireddy and L. Tong, "Exploiting decentralized channel state information for random access," IEEE Trans. Inf. Theory, vol. 51, no. 2, pp. 537-561, Feb. 2005.

[23] V. Naware and L. Tong, "Stability of slotted ALOHA with spatial diversity," in Proc. Int. Conf. Communications, Anchorage, AK, May 2003, pp. $463-467$.

[24] V. Naware, G. Mergen, and L. Tong, "Stability and delay of finite user slotted ALOHA with multipacket reception," IEEE Trans. Inf. Theory, submitted for publication.

[25] S. Hanly and D. Tse, "Power control and capacity of spread-spectrum wireless networks," Automatica, vol. 35, no. 12, pp. 1987-2012, Dec. 1999.

[26] N. Mahravari, "Random-access communication with multiple reception," IEEE Trans. Inf. Theory, vol. 36, no. 3, pp. 614-622, May 1990.

[27] R. Pankaj and A. Viterbi, "Two multi access algorithms for channels with multiple reception capacity," in Proc. TENCON'89. 4th IEEE Region 10 Int. Conf., India, Nov. 1989, pp. 798-803.

[28] G. Mergen and L. Tong, "Stability and capacity of wireless networks with probabilistic receptions: Part I-General topologies," Cornell Univ., Adaptive Communications and Signal Processing Lab., Ithaca, NY, Tech. Rep. ACSP TR-01-03-01, Jan. 2003.

[29] W. Rudin, Real and Complex Analysis. New York: McGraw-Hill, 1986.

[30] L. Tassiulas and A. Ephremides, "Stability properties of constrained queueing systems and scheduling for maximum throughput in multihop radio networks," IEEE Trans. Autom. Control, vol. 37, no. 12, pp. 1936-1949, Dec. 1992.

[31] W. Szpankowski, "Stability conditions for some multiqueue distributed systems: Buffered random access systems," Adv. Appl. Probab., vol. 26, pp. $498-515,1994$. 
[32] B. Tsybakov and W. Mikhailov, "Ergodicity of slotted ALOHA systems," Probl. Inform. Transm., vol. 15, no. 4, p. 301312, Oct.-Dec. 1979.

[33] S. Shakkottai and A. Stolyar, "Scheduling for multiple flows sharing a time-varying channel: The exponential rule," in Translations of the AMS, A Volume in Memory of F. Karpelevich. Providence, R.I.: Amer. Math. Soc., 2002.

[34] M. J. Neely, E. Modiano, and C. Rohrs, "Dynamic power allocation and routing for time varying wireless networks," in Proc. IEEE INFOCOM, San Francisco, CA, Apr. 2003.

[35] S. M. Hedetniemi, S. T. Hedetniemi, and A. L. Liestman, "A survey of gossiping and broadcasting in communication networks," Networks, vol. 18, pp. 319-349, 1988.

[36] B. Hajek, "Information constrained routing in massive ad hoc networks," in Proc. Conf. and Workshop on Stochastic Networks, Madison, WI, Jun. 2000.
[37] D. Aldous and J. Fill, "Reversible Markov chains and random walks on graphs," unpublished manuscript.

[38] R. Ellis, "Discrete Green's functions for products of regular graphs," in Proc. 2001 AMS Nat. Conf., 2001. Invited Talk, Special Session on Graph Theory .

[39] L. Tassiulas, "Scheduling and performance limits of networks with constantly changing topology," IEEE Trans. Inf. Theory, vol. 43, no. 3, pp. 1067-1073, May 1997.

[40] L. Tassiulas and A. Ephremides, "Dynamic server allocation to parallel queues with randomly varying connectivity," IEEE Trans. Inf. Theory, vol. 39, no. 2, pp. 466-478, Mar. 1993. 